\title{
52. DREDGED ROCKS FROM THE ARMORICAN AND CELTIC MARGINS
}

\author{
G. A. Auffret,${ }^{1}$ L. Pastouret,${ }^{1}$ G. Cassat,${ }^{2}$ O. de Charpal,${ }^{3}$ J. Cravatte,${ }^{4}$ and P. Guennoc ${ }^{1}$
}

\section{INTRODUCTION}

This report presents the preliminary results of the study of rocks and sediments obtained by dredging during four cruises of the $\mathrm{R} / \mathrm{V}$ Jean Charcot and $\mathrm{R} / \mathrm{V}$ Le Suroit during a cooperative program between CNEXO and CEPM ( $\mathrm{CH} 58$ : April 1975; SU 01: December 1975; CH 66: February 1976; and $\mathrm{CH}$ 67: March 1976). A bathymetric map of the margins under study, as well as the location of the 27 dredges considered in this report, are presented in Figures 1 and 2 , respectively.

Deep-sea drilling in the Bay of Biscay on Leg 12 (Laughton, Berggren, et al., 1972) and Leg 48 (this volume) and the petroleum industry (Dardel and Rosset, 1971) have provided us with precise (though scattered) data of the geology and stratigraphy of this area.

Our aim is to consider the results from dredged samples in light of the stratigraphic sections recovered from Holes $400 \mathrm{~A}, 401$, and $402 \mathrm{~A}$ of DSDP Leg 48. Data obtained from dredged rocks are of varied value, depending on the condition of their recovery. We have distinguished four rock categories: "in place," "probably in place," "possibly in place," and "not in place."

Rocks of the first category occur in dredges during which wire tension as high as six tons was recorded. Surfaces of these rocks present two aspects: part of the rock is coated with black oxide (manganese?) whereas other surfaces clearly show evidence of breaking. The second and third categories do not present both of these features. Many of these fragments were recovered during dredging; their recovery is characterized by significant increase in the wire tension but not as great as that of the first category. The fourth category includes angular rocks and/or pebbles of varied origins (igneous, metamorphic, sedimentary), often entirely coated by black oxides. They represent different sources, either ice-rafted or from submarine slides. Obviously, information derived from the study of the samples is of varied utility, dependent on the category. The environment of deposition is classified into three categories (Carozzi et al., 1972), consisting of the internal platform (in which we distinguish, where possible, more precise depth ranges), the external platform facies may extend into the bathyal (upper to middle) environment (200 to $1000 \mathrm{~m}$ ?). Location of the dredges, as well as details concerning the dredging operation, are presented in Table 1 . The positions given are those of the vessel at the beginning and end of dredging.

'Centre Océanologique de Bretagne, B.P. 337, 29273 Brest, Cedex, France. 'Société Nationale Elf-Aquitaine, Pau, France.

${ }^{3}$ Institut Francais du Pétrole, B.P. 311, 92502 Rueil-Malmaison, France.

${ }^{4}$ Compagnie Francaise des Pétroles, Bordeaux, France.

\section{PALEOZOIC}

Several dredges on the continental slope of the Goban Spur recovered "granitic" rocks on two morphological structures, Granite Cliff and "Menez Bihan"' (Pautot et al., 1976), in water depths ranging from 3200 to 4200 meters (Figure 3 ). These dredgings also recovered Paleozoic sedimentary and metamorphic rocks on the mid-slope scarp at depths ranging from 2000 to 3000 meters (Pendragon Scarp and "King Arthur Castle").

\section{Granitic rocks (Tables 2 and 3)}

Four dredges on the "Granite Cliff" (SU01-12; CH67-08, 09, 10) and three on the "Menez Bihan" (CH67-11, 12, 13) yielded abundant large blocks of granitic rocks reaching up to 30 centimeters; many of them appear clearly to be "in place," others only "possibly in place." They often are coated by a centimeter-thick layer of ferromanganiferous deposits (Shaaf et al., 1977). The dominant granite facies outcropping on Granite Cliff (CH67-08, 09, 10) is a granodiorite, which we also found on the Menez Bihan (CH67-12). It is an equigranular undeformed rock composed of quartz, andesine, $\mathrm{K}$-feldspar, biotite, and hornblende. In some specimens, it has the composition of a quartz diorite or tonalite, as shown in Table 2 (Didier et al., 1977). The age of this rock, using rubidium-strontium and potassium-argon methods, is 275 m.y.B.P. (total rock) or 290 m.y.B.P. (biotite). According to the radiometric age and petrology, the granodiorite appears to have a close affinity with similar igneous facies which have been described in Iberia (Capdevilla et al., 1973) and attributed to the Variscan intrusive episode which is also found in the nearby continental area (southwest Great Britain, Brittany).

We also recovered, on Granite Cliff (SU-01-12, CH67-10) and on Menez Bihan (CH67-11, 13), other lithologies such as quartz-syenite, leucocratic granite, and two micas, granite or monzonite. In some cases the age of these rocks is identical to the previous ones (SU01-12, D62), or a little younger (CH67-13, 8). Some may represent border facies of the granodioritic intrusive body.

In dredge CH67-18 on the Pendragon Scarp, several angular fragments of lamprophyre were recovered. They are possibly in place or not far from their place of origin. They exhibit a quartz-kersantite facies and have been dated from 207 m.y.B.P. (Table 3); they may have been emplaced during a Permo-Triassic post-orogenic distension episode.

\section{Sedimentary and Metamorpic Rocks}

\section{Metamorphic Rocks (Table 4)}

Two dredges (SU01-11 on the southern flank of "King Arthur Castle" and CH67-18 on the southern end of 


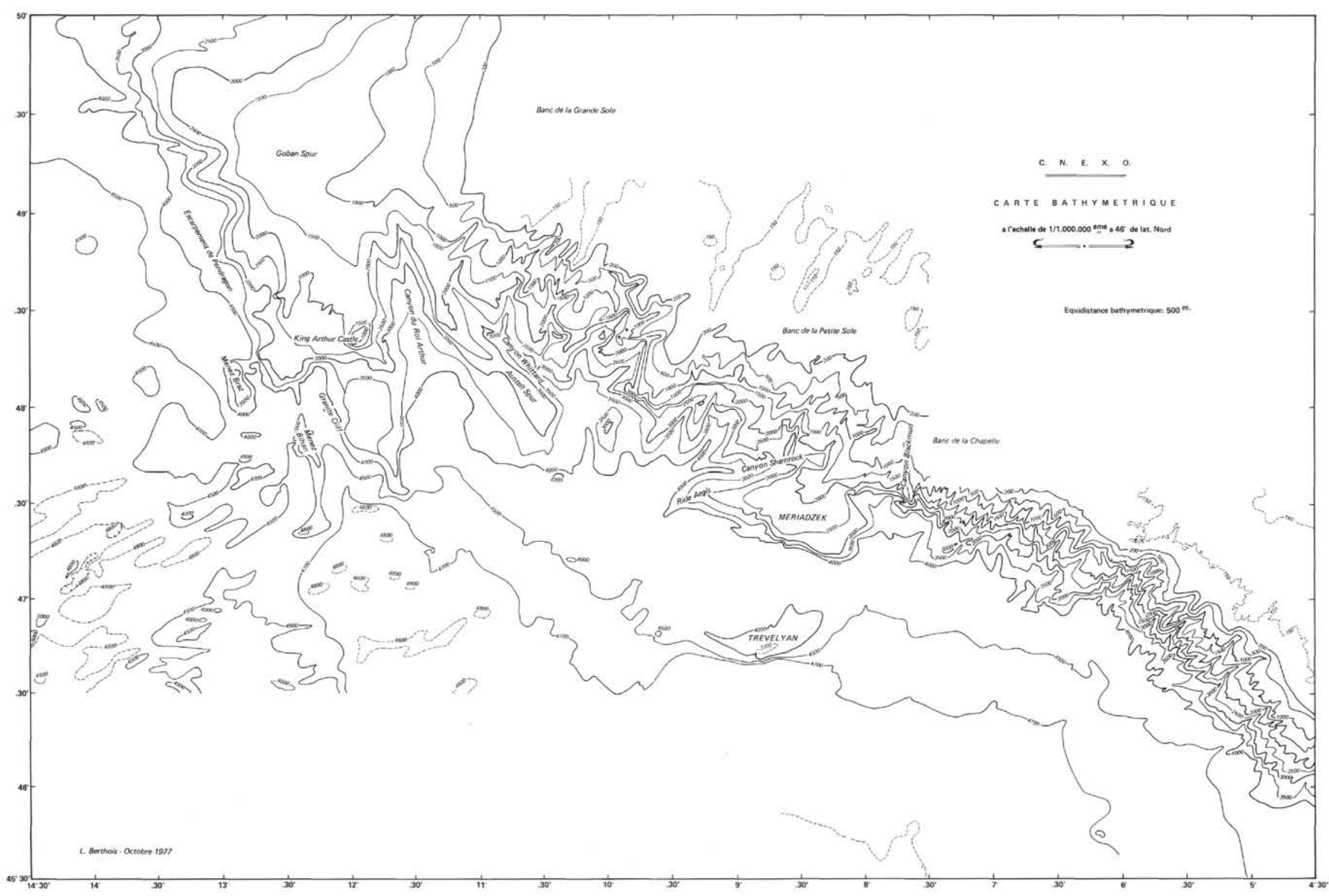

Figure 1. Bathymetric map of Celtic and Armorican margins. 


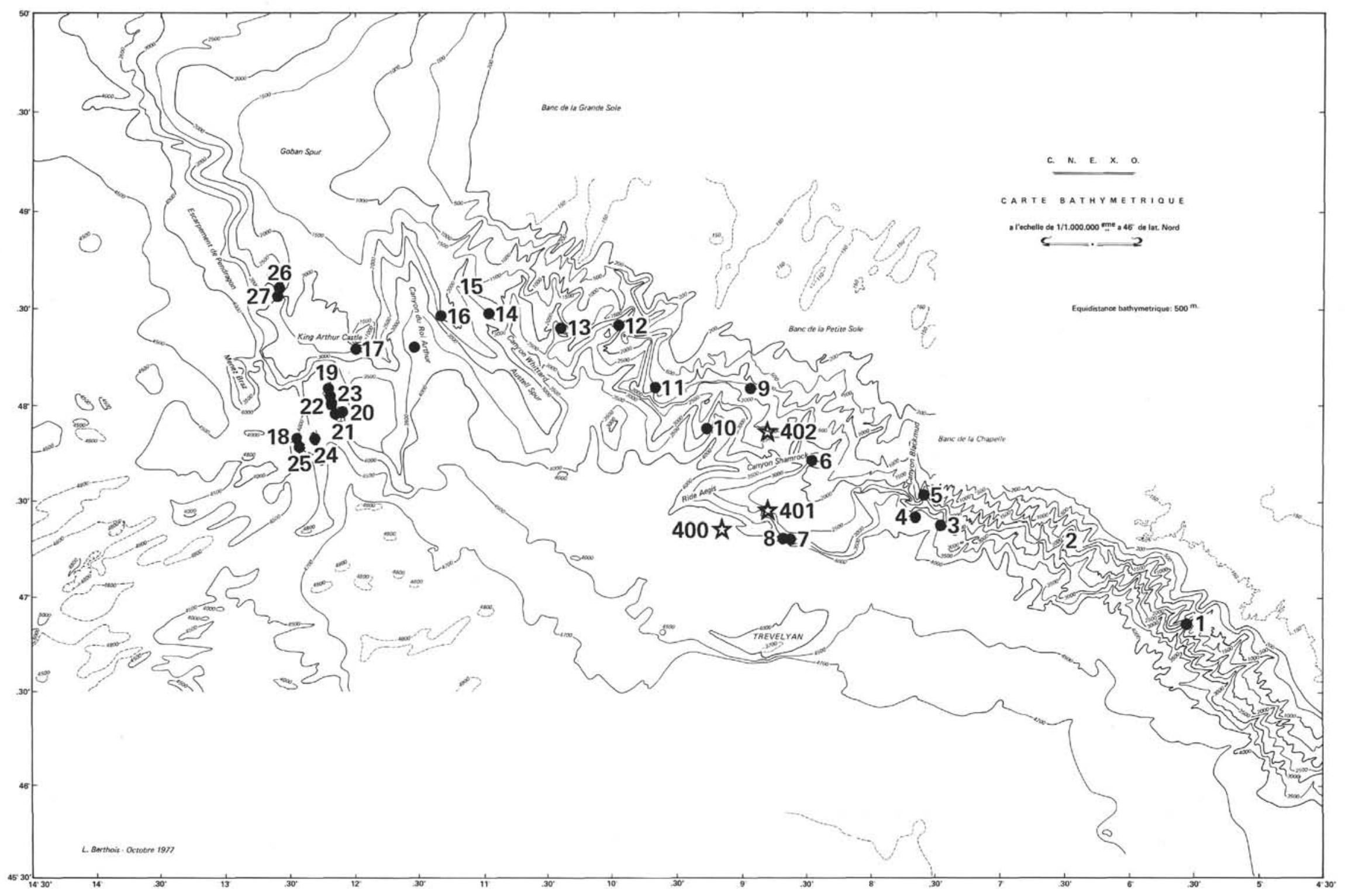

Figure 2. Location of dredges. 1. CH 58-15. 2. CH 58-14. 3. CH 66-06. 4. CH 66-05. 5. CH 58-13. 6. CH 58-09. 7. CH 58-07. 8. CH 66-02. 9. SU 01-01. 10. CH 66-03. 11. SU 01-01. 12. SU 01-16. 13. SU 01-07. 14. SU 01-10. 15. CH 67-21. 16. CH 67-20. 17. SU 01-11. 18. CH 67-11. 19. CH 67-08. 20. SU 0112. 21. $\mathrm{CH}$ 67-10. 22. $\mathrm{CH}$ 67-09. 23. $\mathrm{CH}$ 67-07. 24. $\mathrm{CH}$ 67-12. 25. $\mathrm{CH}$ 67-13. 26. CH 67-18. 27. $\mathrm{CH}$ 67-16. 
TABLE 1

Location of Dredges

\begin{tabular}{|c|c|c|c|c|c|c|}
\hline \multirow[b]{2}{*}{ Dredges } & \multirow{2}{*}{$\begin{array}{c}\text { Number } \\
\text { in } \\
\text { Figure } \\
2\end{array}$} & \multicolumn{2}{|c|}{ Coordinates } & \multicolumn{2}{|c|}{$\begin{array}{l}\text { Water Depth } \\
\text { (m) }\end{array}$} & \multirow[b]{2}{*}{ Tension } \\
\hline & & Position 1 & Position 2 & 1 & 2 & \\
\hline CH 58.01 & & $\begin{array}{l}47^{\circ} 00.1^{\prime} \mathrm{N} \\
06^{\circ} 37.0^{\prime} \mathrm{W}\end{array}$ & $\begin{array}{l}47^{\circ} 01.5^{\prime} \mathrm{N} \\
06^{\circ} 37.5^{\prime} \mathrm{W}\end{array}$ & 3471 & 3236 & Low \\
\hline CH 58-02 & & $\begin{array}{l}47^{\circ} 07.7^{\prime} \mathrm{N} \\
06^{\circ} 36.3^{\prime} \mathrm{W}\end{array}$ & $\begin{array}{l}47^{\circ} 04.2^{\prime} \mathrm{N} \\
06^{\circ} 30.8^{\prime} \mathrm{W}\end{array}$ & 3786 & 3263 & Low \\
\hline CH 58-07 & 7 & $\begin{array}{l}47^{\circ} 16.9^{\prime} \mathrm{N} \\
08^{\circ} 41.2 \mathrm{~W}\end{array}$ & $\begin{array}{l}47^{\circ} 19.0^{\prime} \mathrm{N} \\
08^{\circ} 38.8^{\prime} \mathrm{W}\end{array}$ & 3383 & 3200 & Low \\
\hline CH 58-09 & 6 & $\begin{array}{l}47^{\circ} 43.9^{\circ} \mathrm{N} \\
08^{\circ} 26.7^{\prime} \mathrm{W}\end{array}$ & $\begin{array}{l}47^{\circ} 42.8^{\circ} \mathrm{N} \\
08^{\circ} 26.0^{\prime} \mathrm{W}\end{array}$ & 2231 & 2487 & $\begin{array}{l}\text { High ( } 4 \text { to } 5 \mathrm{~T} \text { ) } \\
\text { at base of slope }\end{array}$ \\
\hline $\mathrm{CH} 58-10$ & & $\begin{array}{l}47^{\circ} 27.9^{\prime} \mathrm{N} \\
07^{\circ} 35.9^{\prime} \mathrm{W}\end{array}$ & $\begin{array}{l}47^{\circ} 30.3^{\prime} \mathrm{N} \\
07^{\circ} 38.0^{\prime} \mathrm{W}\end{array}$ & 2323 & 1280 & Low \\
\hline CH 58-11 & & $\begin{array}{l}47^{\circ} 30.7^{\prime} \mathrm{N} \\
07^{\circ} 41.8^{\prime} \mathrm{W}\end{array}$ & $\begin{array}{l}47^{\circ} 31.3^{\prime} \mathrm{N} \\
07^{\circ} 36.8^{\prime} \mathrm{W}\end{array}$ & 1756 & 1100 & Medium \\
\hline $\mathrm{CH} 58-13$ & 5 & $\begin{array}{l}47^{\circ} 32.6^{\prime} \mathrm{N} \\
07^{\circ} 32.0^{\prime} \mathrm{W}\end{array}$ & $\begin{array}{l}47^{\circ} 32.4^{\prime} \mathrm{N} \\
07^{\circ} 31.3^{\prime} \mathrm{W}\end{array}$ & 1630 & 1575 & $\begin{array}{l}\text { High }(6 \mathrm{~T}) \text { at } \\
\text { base of slope }\end{array}$ \\
\hline CH 58-14 & 2 & $\begin{array}{l}47^{\circ} 15.9^{\prime} \mathrm{N} \\
06^{\circ} 32.2^{\prime} \mathrm{W}\end{array}$ & $\begin{array}{l}47^{\circ} 19.1^{\prime} \mathrm{N} \\
06^{\circ} 32.7^{\prime} \mathrm{W}\end{array}$ & 2496 & 1400 & High $(6 \mathrm{~T})$ \\
\hline CH 58-15 & 1 & $\begin{array}{l}46^{\circ} 47.9^{\prime} \mathrm{N} \\
05^{\circ} 36.2^{\prime} \mathrm{W}\end{array}$ & $\begin{array}{l}46^{\circ} 53.5^{\prime} \mathrm{N} \\
05^{\circ} 34.0^{\prime} \mathrm{W}\end{array}$ & 2140 & 956 & High $(6 \mathrm{~T})$ \\
\hline SU 01-01 & 9 & $\begin{array}{l}48^{\circ} 01.7^{\prime} \mathrm{N} \\
08^{\circ} 59.3^{\prime} \mathrm{W}\end{array}$ & $\begin{array}{l}48^{\circ} 06.0^{\prime} \mathrm{N} \\
08^{\circ} 59.5^{\prime} \mathrm{W}\end{array}$ & 1926 & 1533 & - \\
\hline SU $01-02$ & & $\begin{array}{l}47^{\circ} 58.6^{\prime} \mathrm{N} \\
09^{\circ} 09.5^{\prime} \mathrm{W}\end{array}$ & $\begin{array}{l}48^{\circ} 00.1^{\prime} \mathrm{N} \\
09^{\circ} 09.2^{\prime} \mathrm{W}\end{array}$ & 3100 & 2880 & $\begin{array}{l}\text { High at base of } \\
\text { slope }\end{array}$ \\
\hline SU 01-04 & 11 & $\begin{array}{l}48^{\circ} 00.0^{\prime} \mathrm{N} \\
09^{\circ} 41.2^{\prime} \mathrm{W}\end{array}$ & $\begin{array}{l}48^{\circ} 06.2^{\prime} \mathrm{N} \\
09^{\circ} 40.0^{\prime} \mathrm{W}\end{array}$ & 2800 & 2260 & Medium \\
\hline SU 01-05 & & $\begin{array}{l}48^{\circ} 11.7^{\prime} \mathrm{N} \\
09^{\circ} 55.0^{\prime} \mathrm{W}\end{array}$ & $\begin{array}{l}48^{\circ} 10.5^{\prime} \mathrm{N} \\
09^{\circ} 56.0^{\prime} \mathrm{W}\end{array}$ & & & - \\
\hline SU 01-06 & 12 & $\begin{array}{l}48^{\circ} 24.0^{\prime} \mathrm{N} \\
09^{\circ} 58.5^{\prime} \mathrm{W}\end{array}$ & $\begin{array}{l}48^{\circ} 22.3^{\prime} \mathrm{N} \\
09^{\circ} 57.2^{\prime} \mathrm{W}\end{array}$ & 2700 & 1802 & $\begin{array}{l}\text { High }(6 \mathrm{~T}) \text { at top } \\
\text { of slope }\end{array}$ \\
\hline SU 01-07 & 13 & $\begin{array}{l}48^{\circ} 21.4^{\prime} \mathrm{N} \\
10^{\circ} 24.2^{\prime} \mathrm{W}\end{array}$ & $\begin{array}{l}48^{\circ} 22.3^{\prime} \mathrm{N} \\
10^{\circ} 23.1^{\prime} \mathrm{W}\end{array}$ & 3070 & 2417 & Medium at top \\
\hline SU $01-08$ & & $\begin{array}{l}48^{\circ} 21.6^{\prime} \mathrm{N} \\
10^{\circ} 24.4^{\prime} \mathrm{W}\end{array}$ & $\begin{array}{l}48^{\circ} 20.7^{\prime} \mathrm{N} \\
10^{\circ} 27.8^{\prime} \mathrm{W}\end{array}$ & & & - \\
\hline SU 01-09 & & $\begin{array}{l}48^{\circ} 09.4^{\prime} \mathrm{N} \\
10^{\circ} 10.0^{\prime} \mathrm{W}\end{array}$ & $\begin{array}{l}48^{\circ} 08.5^{\prime} \mathrm{N} \\
10^{\circ} 22.2^{\prime} \mathrm{W}\end{array}$ & 3000 & 2800 & - \\
\hline SU $01-10$ & 14 & $\begin{array}{l}48^{\circ} 26.0^{\prime} \mathrm{N} \\
10^{\circ} 58.2^{\prime} \mathrm{W}\end{array}$ & $\begin{array}{l}48^{\circ} 28.0^{\prime} \mathrm{N} \\
10^{\circ} 54.7^{\prime} \mathrm{W}\end{array}$ & 2910 & 2380 & Medium at top \\
\hline SU 01-11 & 17 & $\begin{array}{l}48^{\circ} 16.0^{\prime} \mathrm{N} \\
12^{\circ} 00.0^{\prime} \mathrm{W}\end{array}$ & $\begin{array}{l}48^{\circ} 19.7^{\prime} \mathrm{N} \\
11^{\circ} 57.5^{\prime} \mathrm{W}\end{array}$ & 3000 & 1423 & $\begin{array}{l}\text { High }(5-6 \mathrm{~T}) \text { at } \\
\text { top }\end{array}$ \\
\hline SU 01-12 & 20 & $\begin{array}{l}47^{\circ} 59.1^{\prime} \mathrm{N} \\
12^{\circ} 04.9^{\prime} \mathrm{W}\end{array}$ & $\begin{array}{l}47^{\circ} 58.5^{\prime} \mathrm{N} \\
12^{\circ} 05.8^{\prime} \mathrm{W}\end{array}$ & 4100 & 3908 & $\begin{array}{l}\text { Medium to high } \\
(6 \mathrm{~T}) \text { at top }\end{array}$ \\
\hline CH 66.01 & & $\begin{array}{l}46^{\circ} 39.8^{\prime} \mathrm{N} \\
08^{\circ} 50.2^{\prime} \mathrm{W}\end{array}$ & $\begin{array}{l}46^{\circ} 42.5^{\prime} \mathrm{N} \\
08^{\circ} 49.5^{\prime} \mathrm{W}\end{array}$ & 4575 & 3713 & - \\
\hline $\mathrm{CH} 66-02$ & 8 & $\begin{array}{l}47^{\circ} 19.0^{\prime} \mathrm{N} \\
08^{\circ} 40.3^{\prime} \mathrm{W}\end{array}$ & $\begin{array}{l}47^{\circ} 21.6^{\prime} \mathrm{N} \\
08^{\circ} 39.1^{\prime} \mathrm{W}\end{array}$ & 3281 & 2438 & $\begin{array}{l}\text { Medium at mid } \\
\text { slope }\end{array}$ \\
\hline $\mathrm{CH}$ 66-03 & 10 & $\begin{array}{l}47^{\circ} 54.0^{\prime} \mathrm{N} \\
09^{\circ} 14.2^{\prime} \mathrm{W}\end{array}$ & $\begin{array}{l}47^{\circ} 53.5^{\prime} \mathrm{N} \\
09^{\circ} 16.8^{\prime} \mathrm{W}\end{array}$ & 3225 & 2944 & High ( 4 to $7 \mathrm{~T})$ \\
\hline $\mathrm{CH} 66-05$ & 4 & $\begin{array}{l}47^{\circ} 26.5^{\prime} \mathrm{N} \\
07^{\circ} 35.0^{\prime} \mathrm{W}\end{array}$ & $\begin{array}{l}47^{\circ} 28.4^{\prime} \mathrm{N} \\
07^{\circ} 35.7^{\prime} \mathrm{W}\end{array}$ & 3038 & 2228 & $\begin{array}{l}\text { High }(6 \mathrm{~T}) \text { at } \\
\text { base of slope }\end{array}$ \\
\hline CH 66-06 & 3 & $\begin{array}{l}47^{\circ} 24.5^{\prime} \mathrm{N} \\
07^{\circ} 29.3^{\prime} \mathrm{W}\end{array}$ & $\begin{array}{l}47^{\circ} 24.8^{\prime} \mathrm{N} \\
07^{\circ} 27.7^{\prime} \mathrm{W}\end{array}$ & 3800 & - & $\begin{array}{l}\text { High ( } 4 \text { to } 5 \mathrm{~T}) \\
\text { at base of slope }\end{array}$ \\
\hline $\mathrm{CH}$ 67-07 & 23 & $\begin{array}{l}47^{\circ} 59.8^{\prime} \mathrm{N} \\
12^{\circ} 08.4^{\prime} \mathrm{W}\end{array}$ & $\begin{array}{l}48^{\circ} 00.8^{\prime} \mathrm{N} \\
12^{\circ} 02.6^{\prime} \mathrm{W}\end{array}$ & 3320 & 3506 & High $(7 \mathrm{~T})$ at top \\
\hline CH 67.08 & 19 & $\begin{array}{l}47^{\circ} 59.8^{\prime} \mathrm{N} \\
12^{\circ} 07.7^{\prime} \mathrm{W}\end{array}$ & $\begin{array}{l}47^{\circ} 59.7^{\prime} \mathrm{N} \\
12^{\circ} 04.4^{\prime} \mathrm{W}\end{array}$ & 3365 & 3353 & High \\
\hline CH 67-09 & 22 & $\begin{array}{l}47^{\circ} 58.9^{\prime} \mathrm{N} \\
12^{\circ} 06.8^{\prime} \mathrm{W}\end{array}$ & $\begin{array}{l}47^{\circ} 59.0^{\prime} \mathrm{N} \\
12^{\circ} 02.5^{\prime} \mathrm{W}\end{array}$ & 3500 & & $\begin{array}{l}\text { High }(3 \text { to } 4 \mathrm{~T}) \text { a } \\
\text { at base of slope }\end{array}$ \\
\hline $\mathrm{CH} 67-10$ & 21 & $\begin{array}{l}47^{\circ} 59.2^{\prime} \mathrm{N} \\
12^{\circ} 07.1^{\prime} \mathrm{W}\end{array}$ & $\begin{array}{l}48^{\circ} 00.3^{\prime} \mathrm{N} \\
12^{\circ} 04.8^{\prime} \mathrm{W}\end{array}$ & 3411 & 3365 & $\begin{array}{l}\text { High at mid- } \\
\text { slope }\end{array}$ \\
\hline $\mathrm{CH} 67-12$ & 24 & $\begin{array}{l}47^{\circ} 47.5^{\prime} \mathrm{N} \\
12^{\circ} 16.2^{\prime} \mathrm{W}\end{array}$ & - & 4141 & 3500 & Medium at top \\
\hline $\mathrm{CH} 67-13$ & 25 & $\begin{array}{l}47^{\circ} 46.0^{\prime} \mathrm{N} \\
12^{\circ} 19.6^{\prime} \mathrm{W}\end{array}$ & $\begin{array}{l}47^{\circ} 44.5^{\prime} \mathrm{N} \\
12^{\circ} 21.2^{\prime} \mathrm{W}\end{array}$ & 4152 & 3940 & High $(6$ to $7 \mathrm{~T})$ \\
\hline $\mathrm{CH} 67-16$ & 27 & $\begin{array}{l}48^{\circ} 34.2^{\prime} \mathrm{N} \\
12^{\circ} 35.1^{\prime} \mathrm{W}\end{array}$ & $\begin{array}{l}48^{\circ} 36.0^{\prime} \mathrm{N} \\
12^{\circ} 33.6^{\prime} \mathrm{W}\end{array}$ & 3040 & 2090 & High $(7 \mathrm{~T})$ at top \\
\hline $\mathrm{CH} 67-18$ & 26 & $\begin{array}{l}48^{\circ} 34.8^{\prime} \mathrm{N} \\
12^{\circ} 34.3^{\prime} \mathrm{W}\end{array}$ & $\begin{array}{l}48^{\circ} 34.5^{\prime} \mathrm{N} \\
12^{\circ} 32.5^{\prime} \mathrm{W}\end{array}$ & 2050 & 2120 & High \\
\hline $\mathrm{CH} 67.20$ & 16 & $\begin{array}{l}48^{\circ} 26.6^{\prime} \mathrm{N} \\
11^{\circ} 19.9^{\prime} \mathrm{W}\end{array}$ & ? & 2344 & & - \\
\hline $\mathrm{CH} 67.21$ & 15 & $\begin{array}{l}48^{\circ} 36.3^{\prime} \mathrm{N} \\
11^{\circ} 10.1^{\prime} \mathrm{W}\end{array}$ & $\begin{array}{l}48^{\circ} 35.4^{\prime} \mathrm{N} \\
11^{\circ} 15.1^{\prime} \mathrm{W}\end{array}$ & 2738 & 2104 & $\begin{array}{l}\text { High }(4 \mathrm{~T}) \text { at } \\
\text { mid slope }\end{array}$ \\
\hline $\mathrm{CH} 67-11$ & 18 & $\begin{array}{l}47^{\circ} 46.9^{\prime} \mathrm{N} \\
12^{\circ} 18.4^{\prime} \mathrm{W}\end{array}$ & $\begin{array}{l}47^{\circ} 45.2^{\prime} \mathrm{N} \\
12^{\circ} 20.3^{\prime} \mathrm{W}\end{array}$ & 4200 & 3590 & - \\
\hline
\end{tabular}

Pendragon Scarp) have yielded abundant sedimentary and metamorphic pre-Mesozoic rocks (Table 4).

In SU01-11, we recovered ("possibly in place") many fragments of gray to pale green chloritic schist that display S0 stratification, a non-parallel S1 schistosity, and are characterized by a low-grade metamorphism (anchizone or epizone of Kübler, 1968). We also observed small fragments of schists of similar facies in Upper Cretaceous to Eocene conglomerates.

In CH67-18, abundant small schist debris is characterized by a primary stratification S0, alternately underlain by fine phyllitic beds (mainly chlorite and sericite) and coarser beds of quartz, chlorite, scarce feldspars, and lithic fragments. The stratification S0 and the schistosity S1 (parallel or not to S0) are crossed by a broad crenulation cleavage (S2). These schists have been metamorphosed in the epizone (Kübler, 1968). In dredge SU01-11, we also found abundant fragments of mica schist included in a clayey matrix. On Granite Cliff and Menez Bihan, angular or rounded fragments or blocks of high-rank metamorphic rocks were recovered. All the "granulite facies" of these rocks have been described as granulites and charnockites (Didier et al., 1977).

\section{Sedimentary Rocks (Tables $\mathbf{4}$ and $\mathbf{5}$ )}

In dredge CH67-18 (possibly in place), we recovered abundant rounded or angular fragments of shallow-water biomicritic dark limestone that included echinoderms, bryozoans, algae, trilobites, brachiopods, gastropods, and ostracodes. The association suggests a Carboniferous (Visean) age. Pebbles or angular fragments of rocks of the same age have been also found at Sites CH67-08 and $\mathrm{CH} 67-10$. Abundant angular fragments and large blocks (up to $30 \mathrm{~cm}$ ) of pale green sandstone have been also recovered ("in place") at Site CH67-18. According to Krynine (1948), these fragments are low-rank graywacke or quartz-wacke (Dott, 1964). In the same dredge, subangular fragments of layered green and gray sandstones have also been sampled. The angular grains (60 to $150 \mu \mathrm{m}$ ) consist dominantly of quartz and plagioclase, bound by a fine calcitic or phyllitic cement. A pre-Mesozoic age for these facies (upper? Paleozoic), although very probable, is not definitely demonstrated.

\section{JURASSIC (Table 6)}

Jurassic sediments (Kimmeridgian) were encountered at Site 401 only, 255 meters sub-bottom (Dupeuble, this volume). It comprises 47.5 meters of white limestone, the microfacies of which are characterized by the presence of algae, foraminifers, echinoderms, pelecypods debris, pellets, oolites, and coral fragments embedded in a finegrained matrix. The microfacies suggest a shallow-water (perireefal) environment.

Jurassic rocks were recovered only in two dredges, although they were not obtained "in place." The indication of these facies appear noteworthy. Sample CH67-07-C4 (Plate 1 is an angular pebble of pinkish white intrabiomicrite limestone, that includes algae (Paleodasycladus mediterraneus, Macroporella sp.), echinoderms, gastropods, pelecypods, and small foraminifers. The microfacies suggest a 


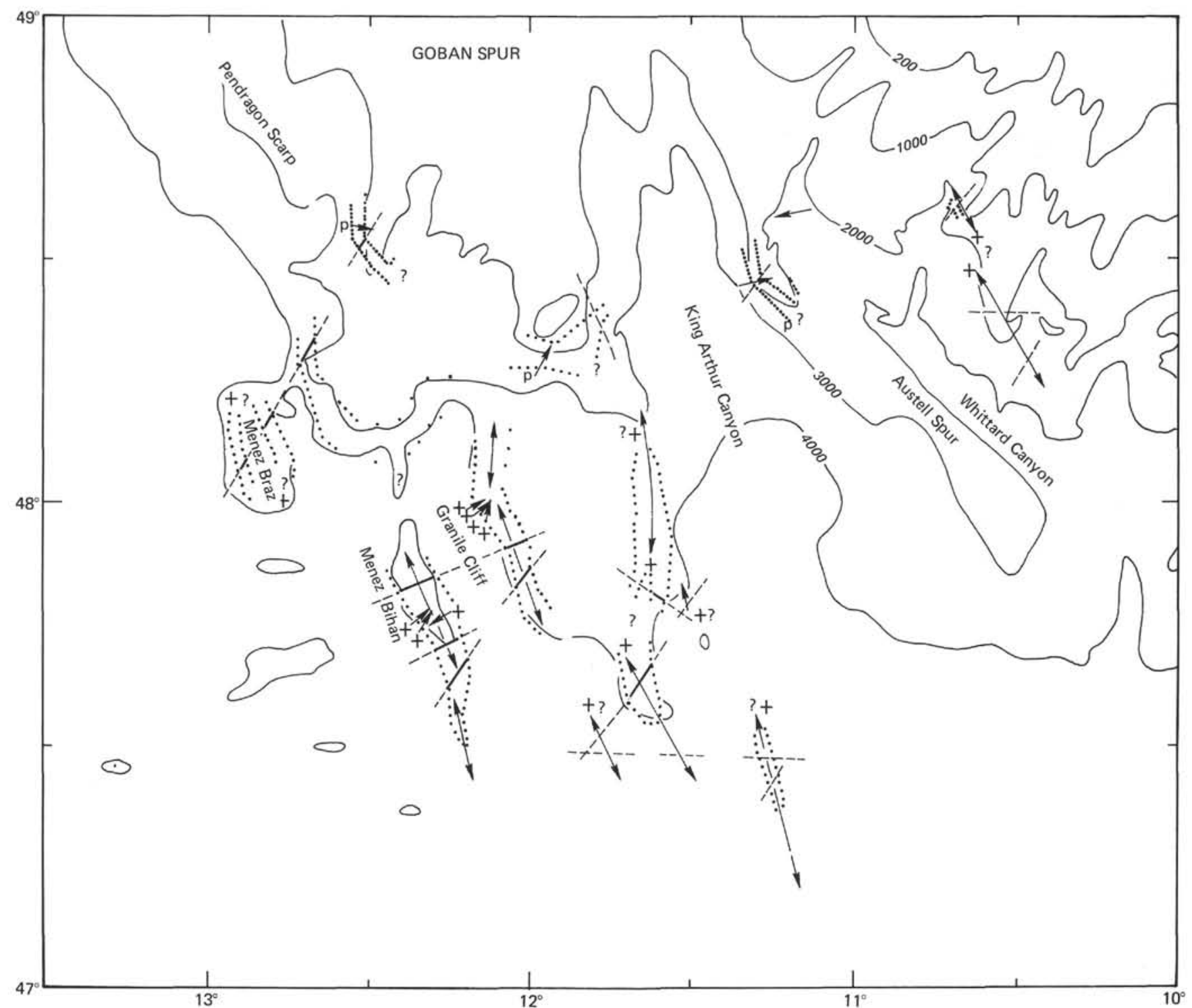

Figure 3. Pre-Mesozoic basement outcrops areas on the southwest continental slope of Goban Spur. Basement outcrops (.....) deduced from dredges $(\rightarrow)(+:$ granitic rocks recovered, P:paleozoic rocks recovered).Basement highs $(\longrightarrow+$ ?), possibly granitic intrusions, deduced from seismic reflection profiles (---).

Late Jurassic (Kimmeridgian, Portlandian) intertidal (lagoonal) environment. This formation is superimposed on the granitic basement. At SU01-01, two fragments of Upper Jurassic dolomitic $(6 \%$ limestones $(\mathrm{C} 1)$ have been recovered. These are dark colored, bored, brachiopod-bearing, micrite limestones, that contain scarce ostracodes, pelecypods, and Globochaet (?) debris. It is a microfacies common in the Upper Jurassic, and supposedly is characteristic of the external platform (upper bathyal?). Although the origin of this sample is probably allochthonous, the fact that two pieces of similar facies were recovered suggests they may have been displaced only a short distance from their origin.

Pastouret and Auffret (1976) have reported the occurrence of calpionellid limestone outcrops on the scarp of the Meriadzek Terrace. These limestones are characterized by a micritic matrix, in which are abundant
Calpionellidae, an association characteristic of the upper Tithonian (Remane, 1974). In the same dredge were clasts of the same formation in a biomicrosparitic, pseudo-oolitic limestone of Late Jurassic to Early Cretaceous age. The occurrence of calpionellid limestone in the Bay of Biscay also has been reported: on the Aquitanian continental shelf at the site of Cormoran I (Dardel and Rosset, 1971), on the Banc Le Danois of the Spanish continental margin (Boillot et al., 1971; Durand-Delga, 1973), and west of Iberia on the Galicia Bank (Dupeuble et al., 1976).

Upper Jurassic and Lower Cretaceous calpionellid limestones are a common facies in the Tethyan environment from Europe to the Caribbean. They are considered indicative of a "relatively deep environment." The reliability of this facies as an indication of depth is highly questionable; they could be present in both deep-water and shallow-water environments. In the latter case, however, 
TABLE 2

Modal and Chemical Analysis of Fresh Samples of Granodioritic Rocks (Didier et al., 1977)

\begin{tabular}{|c|c|c|c|c|c|c|c|c|c|c|c|c|}
\hline \multirow{3}{*}{ CH 6708 D 1} & \multicolumn{2}{|c|}{ Quartz } & \multicolumn{2}{|c|}{ Plagioclase } & \multicolumn{2}{|c|}{ K-Feldspar } & \multicolumn{2}{|c|}{ Biotite } & \multicolumn{2}{|c|}{ Hornblende } & \multicolumn{2}{|c|}{$\begin{array}{c}\text { Accessory } \\
\text { Minerals }\end{array}$} \\
\hline & \multicolumn{2}{|c|}{$20-25$} & \multicolumn{2}{|l|}{$35-55$} & \multicolumn{2}{|c|}{$1-15$} & \multicolumn{2}{|c|}{20} & \multicolumn{2}{|c|}{-} & \multicolumn{2}{|c|}{$\sim 1 \%$} \\
\hline & $\mathrm{SiO}_{2}$ & $\mathrm{Al}_{2} \mathrm{O}_{3}$ & $\mathrm{Fe}_{2} \mathrm{O}_{3}$ & $\mathrm{FeO}$ & $\mathrm{MgO}$ & $\mathrm{CaO}$ & $\mathrm{Na}_{2} \mathrm{O}$ & $\mathrm{K}_{2} \mathrm{O}$ & $\mathrm{TiO}_{2}$ & $\mathrm{MnO}$ & $\mathrm{H}_{2} \mathrm{O}^{+}$ & $\mathrm{H}_{2} \mathrm{O}^{-}$ \\
\hline $\mathrm{CH} 6708 \mathrm{D} 1$ & 61.60 & 17.28 & 0.79 & 4.43 & 2.42 & 3.78 & 3.45 & 3.67 & 1.10 & 0.08 & 0.77 & 0.12 \\
\hline CH 6710 D 5 & 62.50 & 16.15 & 0.41 & 3.90 & 3.54 & 3.25 & 3.62 & 3.75 & 0.72 & 0.08 & 1.39 & 0.17 \\
\hline
\end{tabular}

TABLE 3

Igneous Rocks Dredged on the Continental Slope of the Goban Spur Area

\begin{tabular}{|c|c|c|c|c|}
\hline Sample & Nature & Recovery & Radiometric Age ${ }^{\mathrm{a}}$ (m.y.) & Observations \\
\hline SU 0112 D 61 & Quartz-syenite & Angular fragment & & \\
\hline $12 \mathrm{D} 62$ & Two-mica granite & Angular fragment & $274 \pm 7$ (T.R. Rb/Sr) & \\
\hline $\mathrm{CH} 6708 \mathrm{D} 1$ & Granodiorite & 20 to $30 \mathrm{~cm}$ size fragments & $\begin{array}{l}275 \pm 3(\mathrm{~T} . \mathrm{R} . \mathrm{Rb} / \mathrm{Sr}) \\
281(\mathrm{Bi}, \mathrm{Rb} / \mathrm{Sr})^{*}\end{array}$ & \\
\hline $09 \mathrm{D} 4$ & Quartz diorite & Angular fragment & & \\
\hline $10 \mathrm{D} 51$ & Quartz diorite & Fragments & $270 \pm 3$ (T.R. $\mathrm{Rb} / \mathrm{Sr}$ ) & \\
\hline D 54 & & Large size ( $>10 \mathrm{~cm}$ long) & $290(\mathrm{Bi}, \mathrm{K} / \mathrm{Ar})$ & \\
\hline $10 \mathrm{D} 52$ & Quartz syenite & Broken fragment & & $\begin{array}{l}\text { Pinkish rock, myrmekite, and } \\
\text { perthite }\end{array}$ \\
\hline $11 \mathrm{D} 3$ & Monzonite & Angular fragments & & Gray monzonite or monzo-diorite \\
\hline $12 \mathrm{D} 7$ & Granodiorite & Angular fragments & & $\begin{array}{l}\text { Weathered and covered with Fe-Mn } \\
\text { crust }\end{array}$ \\
\hline $13 \mathrm{D} 8$ & Granite & Broken blocks & $\begin{array}{l}251 \pm 2 \text { (T.R. Rb/Sr) } \\
207(\mathrm{Bi}, \mathrm{K} / \mathrm{Ar})\end{array}$ & Granite - syenite \\
\hline $13 \mathrm{D} 9$ & Leucogranite & & & $\begin{array}{l}\text { Cataclastic rock with aplitic zone, } \\
\text { grenat and scarce biotite }\end{array}$ \\
\hline $13 \mathrm{D} 10$ & Potassic granite & Angular fragments & & $\begin{array}{l}\text { Fine-grained, gray-green cataclastic } \\
\text { rocks, myrmekites and perthites }\end{array}$ \\
\hline $18 \mathrm{G} 10$ & Lamprophyre: & $\begin{array}{l}\text { Several similar pinkish } \\
\text { "quartz-kersantite" angular } \\
\text { fragments }(5 \mathrm{~cm}) \text { possibly } \\
\text { in place }\end{array}$ & $207(\mathrm{Bi} \mathrm{K} / \mathrm{Ar})$ & $\begin{array}{l}\text { Plagioclase and abundant biotite, } \\
\text { calcite, secondary chlorite }\end{array}$ \\
\hline
\end{tabular}

${ }^{a}$ Radiometric determinations were made at Société Nationale Elf-Aquitaine (SNEAP) Pau, France, by I. Esquevin; and at Université de Rennes, France, by Dr. P. Vidal (*).

calpionellids are generally rather badly preserved (Durand-Delga, personal communication). The scarce data we obtained, and the indication of different sources, suggest that a carbonate platform of Tethyan affinity, characterized by intertidal to upper bathyal water depth, prevailed during the Late Jurassic and extended on the Celtic margin as far as 48 degrees north.

\section{LOWER CRETACEOUS}

During Leg 48, Lower Cretaceous deposits were recovered at the three sites. At Site 401, Neocomian reddish shallow-water limestone contains fragments of algae, bryozoans, Trocholina, corals, echinoderms, and sponge spicules. This is overlain by a 5-cm-thick layer of Aptian chalk. Lower Aptian clayey limestones with scarce small planktonic and benthic foraminifers were recovered at Site
402. They are overlain by upper Aptian and Albian dark marly chalk and carbonaceous calcareous mudstone, the benthic foraminiferal microfauna of which indicate deposition in shallow water. Upper Aptian to upper Albian sediments at Site 400 consist of a sequence of alternating light gray marly chalk and black carbonaceous calcareous mudstone that include radiolarians and rare planktonic foraminifers. The benthic foraminiferal microfauna does not contain aragonitic species of two assemblages, one from shallow-water origin and one of presumed deep water affinity (upper bathyal or deeper). These are overlain by Campanian chalk, thus implying an approximately 30-m.y. hiatus. An even longer hiatus occurs at Site 401 between Aptian and Campanian chalks. At Site 402, the Lower Cretaceous section ends with Albian sponge spicule-bearing claystone. Calcisphere and abundant Pithonella ovalis have 
TABLE 4

Paleozoic Sandstones and Metamorphic Rocks

\begin{tabular}{|c|c|c|c|c|}
\hline Sample & Nature & Recovery & Age & Observations \\
\hline SU 0111 D 3 & Chlorite-schist & $\begin{array}{l}\text { Gray angular fragments possibly } \\
\text { in place }\end{array}$ & Paleozoic (?) & $\begin{array}{l}\text { Schistosity S1 non-parallel to stratifi- } \\
\text { cation So }\end{array}$ \\
\hline $\mathrm{CH} 6718 \mathrm{G} 7$ & Sandstone & $\begin{array}{l}\text { Several pale green angular frag- } \\
\text { ments of medium size }(10 \mathrm{~cm} \text { ) } \\
\text { and one large block (up to } 30 \\
\mathrm{~cm} \text { ) in place }\end{array}$ & Paleozoic (?) & $\begin{array}{l}\text { Homogeneous very fine grained rock. } \\
\text { "Quartz-wacke" "low rank gray- } \\
\text { wacke"" }\end{array}$ \\
\hline CH 6718 PG & Schists & $\begin{array}{l}\text { Numerous gray schists debris of } \\
\text { small }(5 \mathrm{~cm}) \text { or very small }(2 \text { to } \\
3 \mathrm{~cm}) \text { in size }\end{array}$ & Paleozoic (?) & Low-grade (epizone) metamorphic rocks \\
\hline CH 671869 & Sandstone & $\begin{array}{l}\text { Several sub-angular fragments of } \\
\text { gray and green sandstone with } \\
\text { centimetric bedding }\end{array}$ & ? & \\
\hline SU $0111 \mathrm{D} 1$ & Micaschist & $\begin{array}{l}\text { Numerous gray fragments poss- } \\
\text { ibly in place or reworked in a } \\
\text { breccia }\end{array}$ & ? & $\begin{array}{l}\text { Biotite (chloritized), muscovite, } \\
\text { kyanite, apatite, garnet }\end{array}$ \\
\hline
\end{tabular}

TABLE 5

Paleozoic Sedimentary Rocks

\begin{tabular}{|c|c|c|c|c|c|}
\hline Sample & Microfacies & Recovery & Fauna & Environment & Age \\
\hline $\mathrm{CH} 6708 \mathrm{C} 6$ & Biomicritic limestone & $\begin{array}{l}\text { Dark angular fragments } \\
\text { possibly in place }\end{array}$ & $\begin{array}{l}\text { Echinoderms, bryozoans, } \\
\text { foraminifers }\end{array}$ & $\begin{array}{l}\text { Shallow water } \\
\text { Internal platform }\end{array}$ & Carboniferous \\
\hline CH 6708 B 84 & $\begin{array}{l}\text { Intrabiomicrosparitic } \\
\text { limestone }\end{array}$ & Rounded pebble & $\begin{array}{l}\text { Algae, echinoderms, benthic } \\
\text { foraminifers, gastropods, } \\
\text { brachiopods }\end{array}$ & $\begin{array}{l}\text { Shallow water } \\
\text { Internal platform }\end{array}$ & $\begin{array}{l}\text { Lower carboniferous } \\
\text { (Visean) }\end{array}$ \\
\hline $\mathrm{CH} 6708 \mathrm{C} 9$ & Biomicritic limestone & Gray rounded fragment & $\begin{array}{l}\text { Pelecypods, ostracodes, } \\
\text { echinoderms, trilobites, } \\
\text { cephalopods }\end{array}$ & Platform & Paleozoic \\
\hline CH 6710 B 172 & $\begin{array}{l}\text { Biopelmicritic or } \\
\text { sparitic limestone }\end{array}$ & - & $\begin{array}{l}\text { Algae, echinoderms, and } \\
\text { foraminifers }\end{array}$ & Internal platform & Carboniferous \\
\hline $\mathrm{CH} 6710$ B 173 & $\begin{array}{l}\text { Silty biopelmicritic } \\
\text { limestone }\end{array}$ & - & $\begin{array}{l}\text { Mollusks (gastropods) } \\
\text { Serpulidae }\end{array}$ & Shallow water & $\begin{array}{l}\text { Paleozoic (?) possibly } \\
\text { Visean }\end{array}$ \\
\hline $\mathrm{CH} 6718 \mathrm{C} 12$ & $\begin{array}{l}\text { Argillaceous micritic } \\
\text { limestone }\end{array}$ & $\begin{array}{l}\text { Rounded perforated } \\
\text { block }\end{array}$ & Ostracodes & Lagoonal (?) & Carboniferous (?) \\
\hline CH $6718 \mathrm{C} 182$ & Spongolith & Rounded pebble & $\begin{array}{l}\text { Bryozoans/(Fenestellidae) } \\
\text { echinoderms, brachiopods }\end{array}$ & & Paleozoic (?) \\
\hline $\mathrm{CH} 6718 \mathrm{C} 185$ & $\begin{array}{l}\text { Intrabiomicrosparitic } \\
\text { limestone }\end{array}$ & Dark fragment & $\begin{array}{l}\text { Brachiopods, crinoids, } \\
\text { gastropods, bryozoan debris, } \\
\text { algae, foraminifers }\end{array}$ & & $\begin{array}{l}\text { Carboniferous, } \\
\text { possible Visean }\end{array}$ \\
\hline CH 6718 C 191 & Micritic limestone & Pebble & $\begin{array}{l}\text { Gastropods, ostracodes, and } \\
\text { echinoderms }\end{array}$ & $\begin{array}{l}\text { Platform } \\
\text { (external?) }\end{array}$ & Paleozoic? \\
\hline $\mathrm{CH} 6718 \mathrm{C} 203$ & $\begin{array}{l}\text { Dolomicrosparitic } \\
\text { limestone }\end{array}$ & Pebble & $\begin{array}{l}\text { Scarce echinoderms and } \\
\text { trilobites }\end{array}$ & Shallow water & Paleozoic? \\
\hline $\mathrm{CH} 6718 \mathrm{C} 21$ & $\begin{array}{l}\text { Biopelmicrosparitic } \\
\text { limestone }\end{array}$ & $\begin{array}{l}\text { Two fragments possibly } \\
\text { in place }\end{array}$ & $\begin{array}{l}\text { Small benthic foraminifers, } \\
\text { echinoderm debris, brachio- } \\
\text { pods, cephalopods }\end{array}$ & Internal platform & Carboniferous? \\
\hline $\mathrm{CH} 6718 \mathrm{C} 22$ & $\begin{array}{l}\text { Intrabiomicritic lime- } \\
\text { stone }\end{array}$ & Gray fragment & $\begin{array}{l}\text { Foraminifers, bryozoans, } \\
\text { brachiopods }\end{array}$ & Internal platform & Carboniferous? \\
\hline
\end{tabular}

been also observed in these lithified facies, as well as echinoderm and algal debris in the uppermost levels (Lithophyllum amphiroaeformis).

Lower Cretaceous formations have been abundantly sampled during the dredging operations. It appears that most of the samples are characteristic of neritic reefal or perireefal environment (Plate 1, Figures 2 to 6). Common presence of oxidized colors (iron oxide) suggests a possible emersion. At Site SU01-DR 06, two pebbles of Pithonella-bearing micritic limestone were recovered.

Conglomeratic limestones are also abundant in the region of Granite Cliff and King Arthur Castle. These limestones contain igneous and metamorphic clasts, as well as Lower Cretaceous limestone debris. At Sites SU01-06, CH66-13, and $\mathrm{CH} 58-09$, we also recovered dark carbonaceous marly chalk or mudstone, which is probably the equivalent of the 


\begin{tabular}{|c|c|c|c|c|c|c|}
\hline Stage & Sample & Location & Microfacies & Recov. & Environment & Remarks \\
\hline \multicolumn{7}{|c|}{ LATE JURASSIC TO EARLY CRETACEOUS } \\
\hline Early Cretaceous (?) & $\mathrm{CH} 67 \mathrm{DR} 20 \mathrm{C} 231 / 1$ & Austell Spur & $\begin{array}{l}\text { Biopelsparitic limestone, corals. rudists, algae. bryozoan } \\
\text { echinids }\end{array}$ & o & Reefal environment & Perforated pebble \\
\hline Albian-Cenomanian (?) & $\mathrm{CH} 67 \mathrm{DR} 18 \mathrm{C} 19 / 3$ & Pendragon Escarpment & $\begin{array}{l}\text { Biopelmicritic limestone, Aeolisaccus, Orbitolina. } \\
\text { Dasycladacae, bryozoan, ostracodes, echinids }\end{array}$ & o & Internal platform & Pebble \\
\hline $\begin{array}{l}\text { Late Albian - Early } \\
\text { Cenomanian }\end{array}$ & CH58 DR13 G5 & Chapelle Bank (SW) & Chalk, spiculae, Trocholina, ligneous debris & $\mathrm{xxx}$ & $\begin{array}{l}\text { External platform } \\
\text { (hemipelagic) }\end{array}$ & \\
\hline Albian - Cenomanian & CH58 DR09 B4, B161 & Shamrock Canyon (S) & Silicified mudstone, spiculae, bryozoan, Melobesiae & $\mathrm{xx}$ & Infratidal & $\begin{array}{l}\text { Similar to Vimport facies and } \\
\text { Site } 402\end{array}$ \\
\hline Albian - Cenomanian & CH58 DR09 G1 & Shamrock Canyon (S) & $\begin{array}{l}\text { Carbonaceous calcareous mudstone, silicified micro- } \\
\text { fauna }\end{array}$ & $\mathrm{xxx}$ & $\begin{array}{l}\text { External platform } \\
\text { (hemipelagic) }\end{array}$ & $\begin{array}{l}\text { Similar to carbonaceous mud- } \\
\text { stone Site } 402\end{array}$ \\
\hline Aptian - Cenomanian & CH66 DR03 C24 & Shamrock Canyon (N) & Silicified marly chalk, spiculae, Hedbergella & $\mathrm{x}$ & External platform & \\
\hline Aptian - Cenomanian & CH66 DR03 C23 & Shamrock Canyon (N) & Biomicritic limestone, spiculae, radiolarian, glauconite & $\mathrm{xxx}$ & External platform & \\
\hline Aptian - Albian (?) & SU01 DR06 C102 & Little Sole (SW) & Carbonaceous glauconitic mudstone, spiculae & $\mathrm{xxx}$ & $\begin{array}{l}\text { External platform } \\
\text { (hemipelagic) }\end{array}$ & $\begin{array}{l}\text { Similar to carbonaceous mud- } \\
\text { stone Site } 402\end{array}$ \\
\hline Barremian - Cenomanian & SU01 DR11 G1, G3, G4 & King Arthur Castle & $\begin{array}{l}\text { Breccia with clasts of volcanic rocks and limestone, } \\
\text { rudists, algae, Orbitolina }\end{array}$ & $\mathrm{xxx}$ & Perireefal for clasts & In breccia \\
\hline Aptian & SU01 DR06 C9 & Little Sole (SW) & $\begin{array}{l}\text { Dolomicrosparitic limestone, oncholithe, Lithoco- } \\
\text { dium, Neotrocholina, algal mat }\end{array}$ & $\mathrm{XX}(?)$ & Infratidal & \\
\hline Late Aptian & SU01 DR06 Cl05 & Little Sole (SW) & Silicified micritic limestone, glauconite, Pithonella & 0 & Infralittoral & Pebble (?), similar to Site 402 \\
\hline Late Aptian & CH58 DR13 B18 & Chapelle Bank (SW) & Silicified micritic limestone, glauconite & $\mathrm{xxx}$ & $\begin{array}{l}\text { External platform } \\
\text { (hemipelagic) }\end{array}$ & \\
\hline Aptian & CH58 DR09 G301 & Shamrock Canyon (S) & $\begin{array}{l}\text { Micritic limestone, ligneous debris, Trocholina. } \\
\text { spiculae }\end{array}$ & $\mathrm{xx}$ & External platform & \\
\hline Aptian & CH58 DR09 G6 & Shamrock Canyon (S) & Silicified marly chalk & $\mathrm{xxx}$ & External platform & \\
\hline Early-Mid Barremian & CH58 DR09 G2 & Shamrock Canyon (S) & Micritic limestone, spiculae, Lagenidae & $\mathrm{xxx}$ & $\begin{array}{l}\text { External platform } \\
\text { (hemipelagic) }\end{array}$ & \\
\hline Early Barremian & CH58 DR09 G7 & Shamrock Canyon (S) & Marly chalk, Textulariidae, ligneous debris, glauconite & $\mathrm{xxx}$ & Ext. platform, bathyal & \\
\hline Early Barremian & CH58 DR09 B2, B3 & Shamrock Canyon (S) & Biomicritic limestone, Trocholina, Orbitolina, "corals" & $\mathrm{xxx}$ & Intertidal, infratidal & \\
\hline $\begin{array}{l}\text { Valanginian - } \\
\text { Barremian }\end{array}$ & CH66 DR02 C11-C17 & Meriadzek Escarpment & $\begin{array}{l}\text { Dolomicritic, dolomicrosparitic limestones, algae } \\
\text { (Cayeuxia, Salpingoporella), foram inifers (Choffatella, } \\
\text { Trocholina), rudists, corals, oolites }\end{array}$ & $\mathrm{xxx}$ & Reefal environment & \\
\hline $\begin{array}{l}\text { Hauterivian } \\
\quad(\text { Cenomanian) }\end{array}$ & CH66 DR03 C221 & Shamrock Canyon (N) & $\begin{array}{l}\text { Intrabiopelmicritic limestone, Serpulidae, ostracodes, } \\
\text { spongiae, Trocholina, Aeolisaccus }\end{array}$ & $\mathrm{xx}$ & Infralittoral & $\begin{array}{l}\text { Possibly reworked in } \\
\text { Cenomanian }\end{array}$ \\
\hline $\begin{array}{l}\text { Hauterivian } \\
\quad \text { (Cenomanian) }\end{array}$ & $\mathrm{CH} 67 \mathrm{DR} 07 \mathrm{Cl}$ & Granite Cliff & Dolomicritic limestone, bivalves & $\mathrm{xxx}$ & Lagoonal & $\begin{array}{l}\text { Similar to CH66 DR03 C221, } \\
\text { possibly reworked }\end{array}$ \\
\hline $\begin{array}{l}\text { Valanginian - } \\
\text { Hauterivian }\end{array}$ & CH66 DR02 M2 & Meriadzek Escarpment & $\begin{array}{l}\text { Dolomitic chalk, ostracodes (Cytherella, Macrocypris, } \\
\text { Darwinulina), Epistominidae }\end{array}$ & $\mathrm{xxx}$ & External platform & \\
\hline $\begin{array}{l}\text { Late Jurassic- } \\
\text { Early Cretaceous }\end{array}$ & $\mathrm{CH} 67 \mathrm{DR} 20 \mathrm{C} 22$ & Austell Spur & $\begin{array}{l}\text { Intrabiosparitic limestone, echinids, algae (Clypeina } \\
\text { jurassica), Pfenderina }\end{array}$ & o & Infratidal & Pebble \\
\hline Late Jurassic & SU01 DR01 Cl & Little Sole (SW) & $\begin{array}{l}\text { Dolomicritic limestone, brachiopods, ostracodes, } \\
\text { Globochaetes }\end{array}$ & 0 & External platform & Perforated pebble \\
\hline $\begin{array}{l}\text { Kimmeridgian - } \\
\text { Tithonian }\end{array}$ & CH67 DR07 C4 & Granite Cliff & $\begin{array}{l}\text { Intrabiomicritic limestone, gastropods, foraminifers, } \\
\text { bivalves }\end{array}$ & $\mathrm{o}$ & Intertidal & Perforated pebble \\
\hline \multicolumn{7}{|l|}{ LATE CRETACEOUS } \\
\hline Late Cretaceous & $\mathrm{CH} 67 \mathrm{DR} 20 \mathrm{C} 23$ & Austell Spur & $\begin{array}{l}\text { Micritic limestone, Inoceramus, Globotruncana, radio- } \\
\text { larians, Saccocoma, glauconite }\end{array}$ & o & Bathyal & Perforated pebble \\
\hline
\end{tabular}




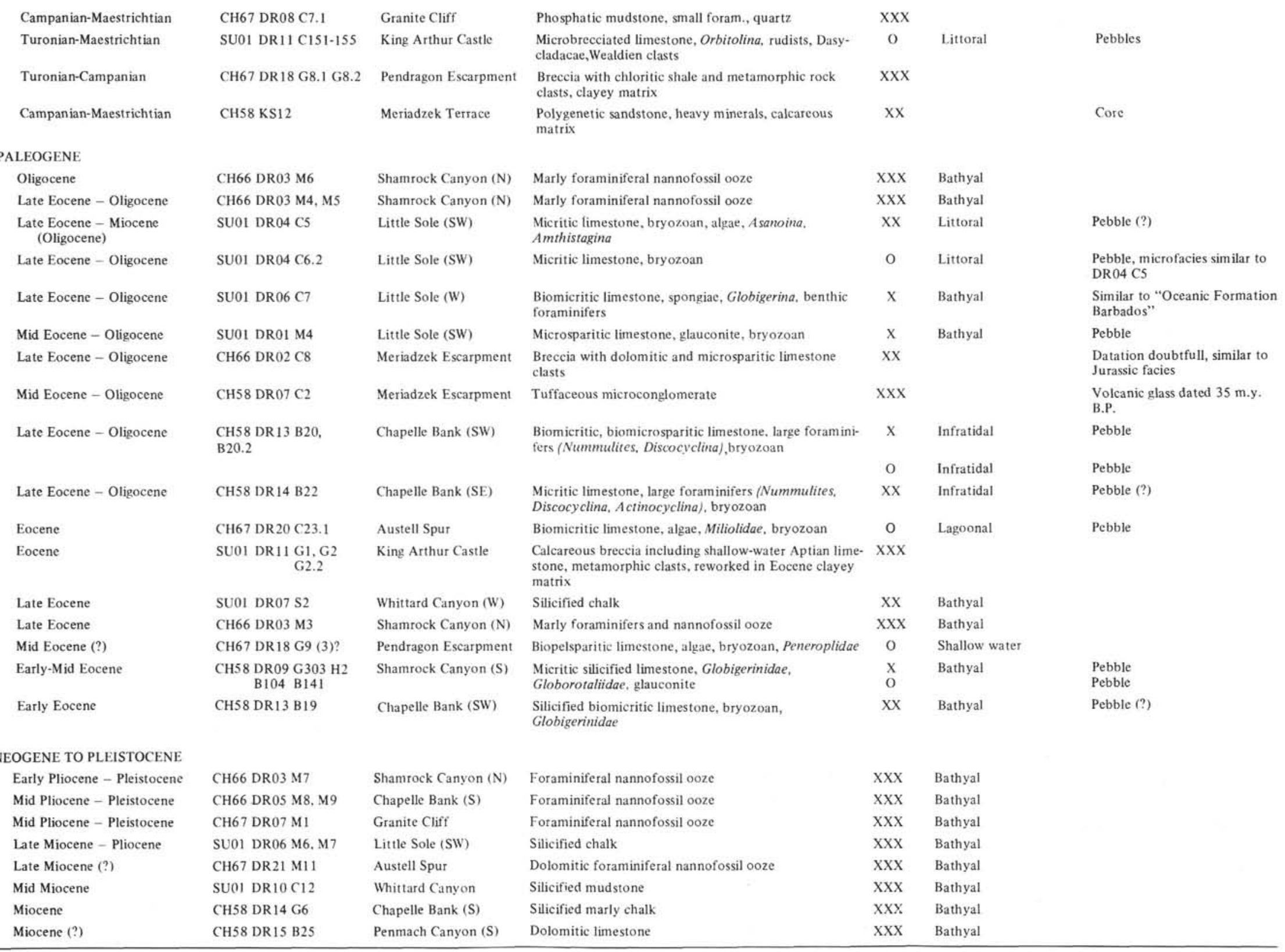


"dark carbonaceous shale" drilled at Site 402. The section dredged at Site CH58-09 (western scarp of the Meriadzek Terrace) suggests an evolution as described below.

In the Barremian, an external platform environment (upper bathyal?) prevailed and continued into middle to late Aptian time when Trocholina-bearing limestone and marly chalk were deposited. In latest Aptian to earliest Albian time, carbonaceous mudstone of a facies similar to the dark shale drilled at Site 402 (Carla Müller, personal communication) were deposited. Finally, limestones containing silicified sponge spicules, and Melobesiae-bearing mudstone (Vimport facies) that might correspond to the Albian limestone and silicified claystone that overlie the dark shale at Site 402 .

It appears that succeeding the deposition of neritic calcareous material from Neocomian to early Aptian, the terrigenous influx increased in the uppermost Aptian and Albian section. The occurrence of algal-bearing Albian limestone at Sites 402, CH58-DR09, and SU01-06 suggests a possible lowering of the sea level by the end of the Early Cretaceous. This is in agreement with the absence of Albian sediment at Site 401 , and the common occurrence of oxidized colors in the Lower Cretaceous limestones.

Pastouret et al. (1974) and Pastouret and Auffret (1976) already have reported the occurrence of rudistid limestone as well as foraminifer and algal-bearing Lower Cretaceous limestone from the Meriadzek Terrace, and discussed the paleogeographical implication of these observations. The development of reef and the extension of perireefal environment in the Early Cretaceous is well known in the Aquitanian Basin, i.e., the Parentis Trough (Dardel and Rosset, 1971) and the northern Pyrenean Trough (Feuillée et al., 1973). The dredged samples indicate a large extension of the carbonate platform. However drilling at Site 400 shows that a deep basin $(2000 \mathrm{~m})$ extended west of this platform. We confirm the occurrence of Aptian-Albian carbonaceous marly chalk or mudstone of shallow-water origin in an area between longitude $8^{\circ} 30^{\prime}$ and $10^{\circ}$ west. Silicified sponge spicules and algal-bearing limestone have been also commonly recovered; they may correspond to some of the uppermost Albian facies drilled at Site 402. Toward the northwest, there is an increasing occurrence of well-lithified conglomeratic facies, some of them including fragments of igneous and metamorphic rocks, as well as Lower Cretaceous limestone debris. Some of the conglomerates could be coeval with the brecciated (but not lithified) facies observed at Site 401 , between the Aptian and Campanian chalk (Bourbon, this volume).

\section{UPPER CRETACEOUS}

White and reddish calcareous chalk and marly chalk of Maestrichtian to late Campanian age represent the Upper Cretaceous at Site 400 . The absence of planktonic foaminifers (probably related to dissolution) and the nature of the benthic foraminifer assemblages indicate a deep-water environment. Foraminifer-bearing reddish chalk of late Campanian to late Maestrichtian age represents the Upper Cretaceous at Site 401. The abundance of planktonic foraminifers and the nature of the benthic foraminiferal assemblage indicate a depth of deposition on the order of
1500 meters, possibly more. No Upper Cretaceous sediments were encountered in Hole 402A.

Upper Cretaceous rocks were dredge-sampled at different sites. At Site CH67-18, limestone breccia was recovered (G81-G82), the matrix of which contains microfauna and nannoflora ranging in age from Late Cretaceous to Eocene.

At Site CH67-08, we sampled many fragments of phosphate-bearing calcareous mudstone (DR08-71; 50\% apatite, $\left.18 \% \mathrm{CaCO}_{3}\right)$ that includes rare sections of small-sized planktonic and benthic foraminifers, as well as intraclasts of the underlying granitic basement (Plate 2, Figure 1). The nannofossil assemblage indicates a Campanian to Maestrichtian age. At Site SU01-11, pebbles of microbrecciated limestone include clasts of quartzite, red claystone (Wealden facies?), Orbitolina debris, rudistids, and algae. The nannofossil assemblage indicate a Late Cretaceous age, but the occurrence of Orbitolina suggests reworking of Lower Cretaceous material. Similar facies have also been recovered "in place"' but these additionally contain a Paleogene nannofossil assemblage.

At Site CH67-DR 20, many fragments (C23/3), of more or less rounded, Upper Cretaceous micritic limestone were recovered (Plate 2, Figure 2). These include abundant Inoceramus prisms, associated with small. pelagic foraminifers (Globotruncanidae, Heterohelicidae), radiolarian spicules, bryozoans, and thin-shelled pelecypods. Another sample is characterized by the occurrence of "Micropithonella" associated with scarce Saccocoma, thin-shelled pelecypods, and Inoceramus prisms. The suggested environment of deposition is the external platform (shelf-break).

Although we did not sample Maestrichtian and Campanian chalks, the condensated sections yielded either clastic or biochemically precipitated material (phosphatic crust) that are in good agreement with the reduced rate of accumulation inferred from the result of the drilling. Drilling at Sites 400 and 401 demonstrated the occurrence of a $30-\mathrm{m} . \mathrm{y}$. hiatus between the uppermost Lower Cretaceous and the Campanian-Maestrichtian deposits. At Site 400 , this follows the deposition of Albian carbonaceous marly chalk at 2000 meters water depth. At Site 401 , it follows the deposition of a thin layer $(5 \mathrm{~cm})$ of Aptian calcareous chalk at a water depth probably less than 500 meters. This sedimentary hiatus is underlain by a conglomeratic mudstone including debris of a phosphatic crust (Bourbon, this volume). The occurrence of a 30-m.y. hiatus between Albian and Campanian chalk at Site 390 from the Blake plateau has also been reported by Benson, Sheridan, et al. (1978). Other examples from deep-sea drilling in the North Atlantic point to the apparent wide extension of this hiatus and its occurrence in a great variety of physiographic conditions. The causality of this hiatus may be on an oceanic scale and related to change in the spreading regime such as increasing accretion rates, in the North Atlantic. This is also the period during which active opening prevails between Europe and Iberia, thus leading to the opening of the Bay of Biscay (Williams, 1975; Olivet et al., 1976). Although the detailed mechanisms leading to the hiatus await further study, it appears that the physiographic situation, tectonic instability and/or active dissolution of the 
carbonate, and low terrigenous supply from the continent should be considered as possible agents. The wide occurrence of conglomeratic facies and phosphatic crusts on the margin suggests that succeeding deposition at a water depth close to sea level by the end of the Early Cretaceous, the environment reached upper bathyal depth by the end of the Late Cretaceous, thus leading to a subsidence of about 500 meters in about 30 m.y. Isotopic measurement (Létolle et al., this volume) suggests a cooling of the surface water during the Late Cretaceous; this should have contributed (with the subsidence) to the interruption of reef development.

\section{PALEOGENE}

At Site 401, lower Paleocene reddish chalk are separated from the Maestrichtian calcareous chalk by a 2-m.y. hiatus. At Site 400 , upper Paleocene chalk, marly chalk, and brown pelagic clay are separated from the Maestrichtian chalk by an 8-m.y. hiatus. The benthic foraminifer species indicate (or at least are compatible with) an abyssal environment. A 3-m.y. hiatus is also observed at Site 401 between lower and middle Paleocene. These chalks are characterized by low accumulation rate $(7 \mathrm{~mm} / 1000 \mathrm{yr})$. Benthic foraminifers indicate a lower bathyal depth of deposition. No Paleocene sediments were encountered at Site 402. At Sites 400 and 401 , an increase in the accumulation rate occurs from early to middle Eocene. Contents in biogenous silica (radiolarian) and terrigenous material also increase. The upper Eocene is lacking at Site 400, while a 1.8-m.y. hiatus is observed between middle and upper Eocene at Site 401. At Site 400, a detailed analysis of a sample from Core 51-3, 43-47 cm (zone P.11, middle Eocene) revealed a rich and diversified fauna of benthic foraminifers which was nearly identical to that described by Beckman (1973) from the middle and upper Eocene of Barbados. The formation of Barbados is considered to represent an abyssal deposit, originally as much as 4000 to 5000 meters deep, thus corresponding to the present depth of Site 400. At Site 401, the composition of the middle Eocene benthic foraminiferal fauna does not change relative to the Paleocene, thus indicating that no bathymetric (or oceanographic) change took place between the Paleocene and early Oligocene. Detailed study of a sample from Zone P.11 (middle Eocene) shows a fauna which appears similar to that of the middle Eocene section of Horizon Guyot (DSDP Site 171), which is thought to have been deposited at a water depth of 1500 to 2300 meters. It thus appears that Site 401 was at its present depth by middle Eocene time. Siliceous marly chalk of late Eocene age were encountered at Site 402 . The benthic foraminiferal microfauna of Core 3 shows the mixing of middle bathyal species and shallow water species. This assemblage could be representative either of the shelf-break ( 200 to $500 \mathrm{~m}$ ) or, assuming that the shallow water species have been displaced, to depths on the order of 1000 to 1500 meters. Because the nannofossil flora has a definite near-shore species composition, as known from some parts of north Germany, we favor the first hypothesis.

Abundant Paleogene rocks have been recovered in our dredging. These are often well-indurated or lithified, thus contrasting with the rather "chalky" appearance of the drilled facies. The information obtained from the study of these rocks is abundant, and sometimes contradictory. The following groups are discussed: the conglomeratic facies, the external platform facies, the internal platform facies, the condensed facies, and finally the volcanic facies from CH58-DR 07.

\section{Conglomerates and Breccias}

These facies were recovered at Sites SU01-11 and CH66-02 (similar facies but devoid of nannofossils from the Paleogene have been recovered at Site CH67-18). At Site SU01-11 (Plate 2, Figure 3) the conglomerates comprise elements of varied sources, metamorphic and igneous basement, and Lower Cretaceous limestone; they are characterized by the occurrence in the matrix of Upper Cretaceous and Eocene nannofossils. At Site CH66-02, the elements of the conglomerate are biomicrosparitic, dolomitic, shallow-water limestone of Jurassic to Early Cretaceous age; the marly phosphate-bearing chalky matrix is of late Eocene to early Oligocene age. These levels appear as condensed sections, but such levels could be the product of different sedimentary processes. Three stages (at least) are necessarily present in the genesis of such facies: (1) ablation of the igneous, metamorphic, or sedimentary clasts from their original formation; (2) transportation from their sources to the depositional area, and (3) lithification. According to the degree of horizontal displacement and the nature of the processes responsible for their reworking (generated by swells, tidal current, or turbidity current), one can consider two hypotheses for the nature of this conglomerate. The first one implies a phase of erosion that started perhaps by the end of the Early Cretaceous, at or near sea level. The erosion products were reworked in an environment with a water depth that was gradually increasing, and finally the clasts were cemented by a fine matrix of hemipelagic origin that includes nannofossils of Late Cretaceous and Eocene-Oligocene ages, all of which may have occurred without large-scale displacement. The second possibility is that during the Late Cretaceous and/or Eocene-Oligocene, the coarse materials resulting from an earlier erosion phase were displaced downslope through mass flow, slides or turbidity currents. It is also possible that one or the other hypothesis may have prevailed on different parts of the margin.

\section{The External Platform Facies}

These facies are apparently the most common and generally characteristic of middle Eocene to Oligocene limestone or silicified chalk sampled east of the $10^{\circ} 30^{\prime} \mathrm{W}$ meridian. At SU01-06, we recovered a late Eocene to Oligocene siliceous chalk that is characterized by a microfauna very similar to the so-called "Barbados oceanic formation" which we reported above as characteristic of the middle Eocene from Site 400. At SU01-07, an upper Eocene white silicified chalk was recovered ("possibly in place'"), characterized by an external platform or deeper facies (upper to lower bathyal?). At SU01-01 ("possibly in place"), middle Eocene to lower Oligocene micritic to microsparitic limestone was recovered which points to an external platform environment of deposition. Similar facies of early to middle Eocene age were sampled at CH58-09 and $\mathrm{CH} 58-13$. 


\section{The Internal Platform Facies}

At CH67-18, we recovered middle Eocene(?) limestone characteristic of high energy shallow-water environment and, at CH67-20, fragments of Eocene algal-bearing limestone suggesting a lagoonal environment (Plate 2, Figure 4); these two facies are not "in place." At SU01-04, ("possibly in place") an algal and Asanoina-bearing limestone (Plate 2, Figure 3) were dredged. This microfacies suggests deposition in the uppermost infratidal zone during late Eocene-Oligocene; a bryozoan-bearing marly limestone of middle Eocene to Oligocene age, suggests a littoral environment. At SU01-01, one sample ("possibly in place") suggests deposition on the external platform, but two samples ("not in place") suggest a littoral environment in the time range from middle Eocene to middle Miocene. At CH58-13, an upper Eocene to Oligocene biomicrosparitic limestone were sampled ("possibly in place") that points to deposition on the uppermost infratidal zone. Another sample ("not in place") of the same age also was deposited in a shallow-water environment. A large foraminifer-bearing limestone from Site CH58-14 ("possibly in place") is also characteristic of deposition in the uppermost infratidal zone during upper Eocene-Oligocene time. A similar facies has been sampled at Site CH58-15.

The recovery of shallow-water limestone of Eocene to Oligocene age has been reported by Pastouret and Auffret (1976). Although it appears that the autochthonous origin is not absolutely convincing, the repetition of this observation merits consideration. One can first postulate that these pebbles were derived from the nearby continental shelf during Oligocene time; another possibility is that they were displaced during the Quaternary, either by a turbidity current or as ice-rafted detritus. A third possibility is that they were derived during the Tertiary from the nearby area of the slope (other than the continental shelf) that had remained shallow. A fourth possibility illustrated at CH58-13 is that, following deposition of chalk on the external platform, an uplift of the margin or a reduction of the water depth through progradation was responsible for the transition from external to internal platform microfacies.

\section{Condensation Level}

At CH66-03, we sampled ("in place") upper Eocene and Oligocene foraminifers and sponge spicules-bearing chalk, but these samples include also lower Miocene, lower Pliocene, and even Quaternary microfauna. The burrowed nature of the sample provides an explanation for this mixing of the fauna, and implies that, since late Eocene, no sediment had been significantly accumulated at this site, pointing to an upper Eocene to Recent hiatus or condensation level. This can be explained either by the presence of strong current preventing deposition, or by oversteepening of the slope in upper Eocene-Oligocene time, or both.

\section{Tuffaceous Conglomerate}

At CH58-07, we sampled ("possibly in place") a conglomeratic tuffaceous conglomerate, including about 30 per cent volcanic glass, rock debris (basalt, limestone), foraminifers, and pteropods. The intraclasts are often rounded and average $50 \mu \mathrm{m}$ in diameter. The microfauna comprises Recent benthic foraminifers characteristic of water depths deeper than 1000 meters. Three nannofossils assemblages are present, characteristic of the Campanian-Maestrichtian, middle Eocene to lower Oligocene, and Quaternary, respectively. A basalt of facies similar to that observed in the conglomerate has an age of 32 m.y.B.P.

No evidence of Cenozoic volcanic activity (except the sample collected at CH58-07) has been observed either in the five holes drilled in the Bay of Biscay, or in the product of our dredges. This puts some doubt on the autochthonous origin of the material recovered. The simultaneous occurrence of Upper Cretaceous and Eocene-Oligocene nannofossils, Quaternary benthic foraminifers, and pteropods, volcanic glass, and basaltic rock debris of Oligocene age, imply the reworking and the mixing of Upper Cretaceous to Quaternary sediments. As a preliminary hypothesis, we propose that this facies results from some kind of slide or mass flow, triggered during the Quaternary by ice-rafted basaltic and volcanic materials.

\section{Conclusion on the Paleogene}

Noteworthy is the absence of Paleocene in the material recovered during the dredging operation. The most common Paleogene facies appears to be the external platform facies, probably equivalent to the upper-lower(?) bathyal siliceous chalk drilled at Site 401 .

This facies has been recovered commonly between $7^{\circ} 30^{\prime} \mathrm{W}$ and $10^{\circ} 30^{\prime} \mathrm{W}$. It is representative of lower Eocene to upper Oligocene samples. Internal platform facies are samples in two areas: west of $10^{\circ} 30^{\prime}$, on the Austell Spur and Pendragon Scarp, and on the Armorican margin east of $7^{\circ} 30^{\prime} \mathrm{W}$. The conglomeratic facies which may be representative of upper bathyal environment was sampled in the area of King Arthur Castle, as well as on the scarp of the Meriadzek Terrace. Consideration of the bathymetric range of the dredge in which Paleogene rock have been sampled suggests that most internal platform facies were recovered in water depth averaging 2000 meters, whereas the external platform facies generally have been sampled deeper. New information is required to clarify this point, which is clearly very important for the subsidence history of the margin.

\section{NEOGENE}

At Site 400, nannofossil chalk and marly nannofossil chalk accumulated at a rate of $20 \mathrm{~mm} / 1000$ years during the Miocene, pointing to a drastic increase of productivity and of the terrigenous supply. The Pliocene rates of accumulation show a further increase to $50 \mathrm{~mm} / 1000$ years. From Core 30 (middle Miocene) upwards, modern abyssal benthic fauna are present. No Miocene nor Pliocene sediment have been recovered at Site 401; at Site 402, Miocene chalk and Pliocene sediment have been recovered in washed core.

At Site SU01-10 ("in place") an indurated and fossil-silicified calcareous glauconite-bearing mudstone was sampled, the nannofossil assemblage of which indicates a middle Miocene age. The suggested environment of deposition is the external platform, i.e., probably upper bathyal. Upper Miocene to Pliocene sediments contain marly chalks such as were recovered at SU01-06, SU01-07, 
CH58-DR 14, and CH58-DR 15. All of these samples indicate deposition, at least on the external platform that is upper to middle bathyal.

The Miocene and Pliocene materials recovered during the dredge operation confirm that at that time most of the continental margin has sunk at least to upper bathyal or (more probably) median to lower bathyal depth. This period also was characterized by very high accumulation rates and progradation to the west of the Aquitaine Basin (Peypouquet, 1977).

\section{CONCLUSION}

Data obtained from dredged rocks allow us to add new information concerning the distribution of facies, and the early stage of the evolution of the Celtic and Armorican margins.

We noted first the occurrence of outcropping plutonic (dominantly granodioritic) bodies on the Celtic margin, that have been intruded into upper Paleozoic shallow-water sedimentary rocks during the late Variscan episode ( 275 to 290 m.y.B.P.). Lamprophyric facies of younger age (207 m.y.B.P.) have been emplaced during a Permo-Triassic post-orogenic distension episode (Olivet et al., 1976). Noteworthy is the apparent lack of Permian, Triassic, and Lower Jurassic sediments. The granitic basement of Granite Cliff and the carboniferous limestone of the Pendragon Scarp are, respectively, overlain by Upper Jurassic limestone and Upper Cretaceous chalk. From Upper Jurassic to at least lower Aptian time, a carbonate platform, characterized by water depth ranging from littoral to at least upper bathyal, stretched from the Aquitaine Basin to Newfoundland. The occurrence of a deep (middle to lower bathyal) and narrow seaway trending southeast-northwest, parallel to this carbonate platform, and related to a first distension phase (Permian to Lias) has been proposed by Olivet et al. (1976). At Site 402, after the deposition of shallow-water carbonaceous mudstone, evidence of shallow depth by the end of the Albian is seen. The absence of Albian deposits at Site 401 , as well as iron oxide impregnation of most of the recovered Lower Cretaceous rocks, suggests reduced water depth on the margin by the end of the Early Cretaceous, while Site 400 was probably already at lower bathyal depth.

The existence of Upper Cretaceous to Eocene conglomeratic facies including clasts of basement rocks (Jurassic and Lower Cretaceous limestone), suggests a phase of erosion that started probably by the end of Early Cretaceous, at or near sea level. This material was reworked and finally cemented at upper bathyal depth in uppermost Cretaceous and/or Paleogene time. Depending on the relative importance of horizontal displacement, two types of evolution can be considered. The first one implies only a few reworkings and progressive cementation by uppermost Cretaceous and Paleogene sediment in an upperslope environment. The second one implies imbedding of the coarse material in a calcareous matrix during a sudden downslope transportation process. It is possible that the coarse debris constituting this conglomerate are in some way related to the erosion surface observed on the seismic profile (Montadert et al., this volume). The reduced accumulation rates that are characteristic of the
Campanian-Maestrichtian have been indirectly confirmed through the observation of two types of condensation levels: either in the form of the previously cited conglomeratic facies, or as phosphatic crusts that have been recovered on the Celtic as well as on the Armorican margin. This crust is probably related to upwelling processes that favored deposition of phosphate-rich material in upper-slope environment. These upwelling processes (probably induced by the wind regime) explain the fertility of the shelf water and the remarkable accumulation of Upper Cretaceous chalk in the channel and surrounding sedimentary basins. The facies of the Paleogene rocks that we recovered confirm that most of the continental slope had already sank by middle-late Eocene, at least to bathyal depth. However, some evidence of internal platform facies in the Eocene and early Oligocene have been observed at water depths of about 2000 meters, particularly on the Celtic margin and on the Armorican margin east of $7^{\circ} 30^{\prime} \mathrm{W}$. Conclusions concerning possible volcanic activity on the Bay of Biscay slope during Oligocene time must be delayed until further observation.

\section{ACKNOWLEDGMENTS}

We wish to acknowledge the collaboration of the Commandant, Officers, and crew of R/V Jean Charcot and R/V Le Surott, on the occasion of the four cruises during which the material of this study has been recovered. We acknowledge also the collaboration of scientists from SNEA: J. Bourroullec and R. Elloy for microfacies observations, J. Aubert for microfauna identification, F. Deres and J. Acheriteguy for nannofossils determination, J. Esquevin and R. Merendy for rock datings, J. C. Lartigou for technical assistance, and, from CFP, R. Brun for microfacies study. We express our thanks to P. Vidal of Rennes University for rock data analysis, to G. Pautot who has been responsible for Cruise CH67, and René Kerbrat for technical assistance during the preparation and execution of this program. P.A. Dupeuble and H.P. Luterbacher helped to improve this manuscript through a critical reading.

\section{REFERENCES}

Beckman, J. P., 1973. Die Foraminiferen der Oceanic Formation (Eocaen-Oligocaen) von Barbados. K1. Antillen: Eclog. Geol. Helv., v. 46, p. 301-412.

Benson, W. E., Sheridan, R. E., et al., 1978. Initial Reports of the Deep Sea Drilling Project, v. 44: Washington (U.S. Government Printing Office).

Boillot, G., Dupeuble, P. A., Durand-Delga, M., et D'Ozouville, L., 1971. Age minimal de l'Atlantique nord d'après la découverte de calcaire tithonique à Calpionelles dans le golfe de Gascogne, C.R. Acad. Sci., Paris., v. 273, p. 671-674.

Capdevilla, R., Corretge, G., and Floor, P., 1973. Les granitöides varisques de la Meseta Ibérique, Bull. Soc. Géol. Fr., p. 209-228.

Carozzi, A. V., Bouroullec, J., Deloffre, R., and Rumeau, J. L., 1972. Microfaciès du Jurassique d'Aquitaine, Bull. Centre de Recherches Pau, SNPA (volume spécial 1).

Dardel, R. A. and Rosset, R., 1971. Histoire géologique et structurale du bassin de Parentis et de son prolongement en mer. Histoire structurale du Golfe de Gascogne: Paris (Technip), v. 4, p. 1-28.

Didier, J., Guennoc, P., and Pautot, G., 1977. Grandiorites, granulites et charnockites de l'Eperon de Goban (marge armoricaine) au contact du domaine océanique, C.R. Acad. Sci. Paris, v. 284D, p. 713-716. 
Dott, R. M., 1964. Wacke, graywacke and matrix. What approach to immature sandstone classification, J. Sediment. Petrol., v. 34 , p. 625-632.

Durand-Delga, M., 1973. Les Calpionelles du golfe de Gascogne, témoin de l'ouverture de l'Atlantique nord, Bull. Soc. Géol. Fr., v. 15 , p. $22-24$

Dupeuble, P. A., Rehault, J. P., Auxietre, J. L., Dunand, J. P., and Pastouret, L., 1976. Résultats de dragages et essais de stratigraphie des bancs de galice, et des montagnes de Porto et de Vigo (marge occidentale ibérique), Marine Geology, v. 22, p. M37-M49.

Feuillée, P., Villanova, M. and Winnock, E., 1973. La dynamique des fosses à turbidites et de leur contenu sédimentaire dans le système pyrénéen, Bull. Soc. Géol. Fr., v. 15 , p. $61-76$.

Krynine, P. D., 1948. The megascopic study and field classification of sedimentary rocks, J. Geology, v. 56, p. 130-165.

Kübler, E. M., 1968. Evaluation quantitative de métamorphisme par la cristallinité de 1'illite, Bull. Centre Rech. Pau SNPA, v. 2, p. 385-397.

Laughton, A. S., Berggren, W. A., et al., 1972. Initial Reports of the Deep Sea Drilling Proejct, v. 12: Washington (U.S. Government Printing Office).

Olivet, J. L., Pastouret, L., Auzende, J. M., and Auffret, G. A., 1976. Armorican margin evolution in relation with Bay of Biscay genesis, Article presented at Colloque IUSGS, Durham.
Pastouret, L. and Auffret, G. A., 1976. Observations sur les microfaciès des roches sédimentaires prélévées sur la marge armoricaine, Rev. Inst. Franc̃ais du Pétrole, v. 31, p. 401-425.

Pastouret,L., Masse, J. P., Phillip, J., and Auffret, G. A., 1974. Sur la présence d'Aptien inférieur à faciès urgonien sur la marge continentale armoricaine, conséquence paléogeographiques, C. R. Acad. Sci., Paris, v. 278, p. 2011-2014. 2011-2014.

Pautot, G., Renard, V., De Charpal, O., Auffret, G. A., and Pastouret, L., 1976. A granite cliff deep on the North Atlantic, Nature, v. 263, p. 669-672.

Peypouquet, J. P., 1977. Les Ostracodes et la connaissance des paléomilieux profonds. Application au Cénozoïque de 1'Atlantique nord-oriental, Thése de Doctorat D'Etat, Bordeaux.

Remane, J., 1974. Les Calpionelles: Publication Université de Geneve.

Shaaf, A., Hoffert, M., Karpoff, A. M., Wirrman, D., 1977. Association de structures stromatolithiques et de foraminiferes sessiles dans un encroûtement ferromanganésifère à coeur granitique en provenance de l'Atlantique nord. C. R. Acad. Sci. Paris, v. 274 D, p. 1705-1708.

Williams, A. C., 1975. Sea-floor spreading in the Bay of Biscay and its relationship to the North Atlantic, Earth Planet. Sci. Lett., v. 24 , p. $440-456$. 



\section{PLATE 1}

Figure $1 \quad C H 67 \mathrm{DR} 07 \mathrm{C4}$ Granite Cliff. Intrabiomicrite, gastropods, mollusks, foraminifers. Kimmeridgian to Tithonian. Intertidal; $15 \times$.

Figure $2 \quad \mathrm{CH} 67 \mathrm{DR} 07 \mathrm{Cl}$ Granite Cliff. Dolomicrosparite, mollusks. Hauterivian (?). Lagoonal; $15 \times$.

Figure $3 \quad C H \quad 66 \quad D R \quad 03 \quad C 22$ Shamrock Canyon (N). Intrabiopelmicrite, partially microsparite, gastropods, ostracodes, spicules, Serpulidae, mollusks. Hauterivian (?). Infralittoral; $12 \times$.

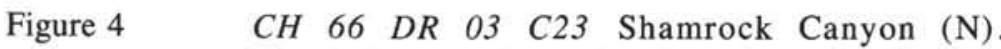
Biomicrite, spicule, radiolarian, glauconite. AptianCenomanian. External platform; $15 \times$.

Figure $5 \quad$ CH 66 DR 02 C16 Meriadzek Escarpment. Biopelmicrite, Rudists, algae, echinoderms, Miliolidae. Valanginian to Barremian. Internal platform, perireefal area; $15 \times$.

Figure $6 \quad C H \quad 67 \quad D R \quad 08 \quad C 71$ Granite Cliff. Phosphatic mudstone, quartz, altered biotite, foraminifers, rare reworked Hedbergella. Campanian-Maestrichtian. $12 \times$. 
PLATE 1
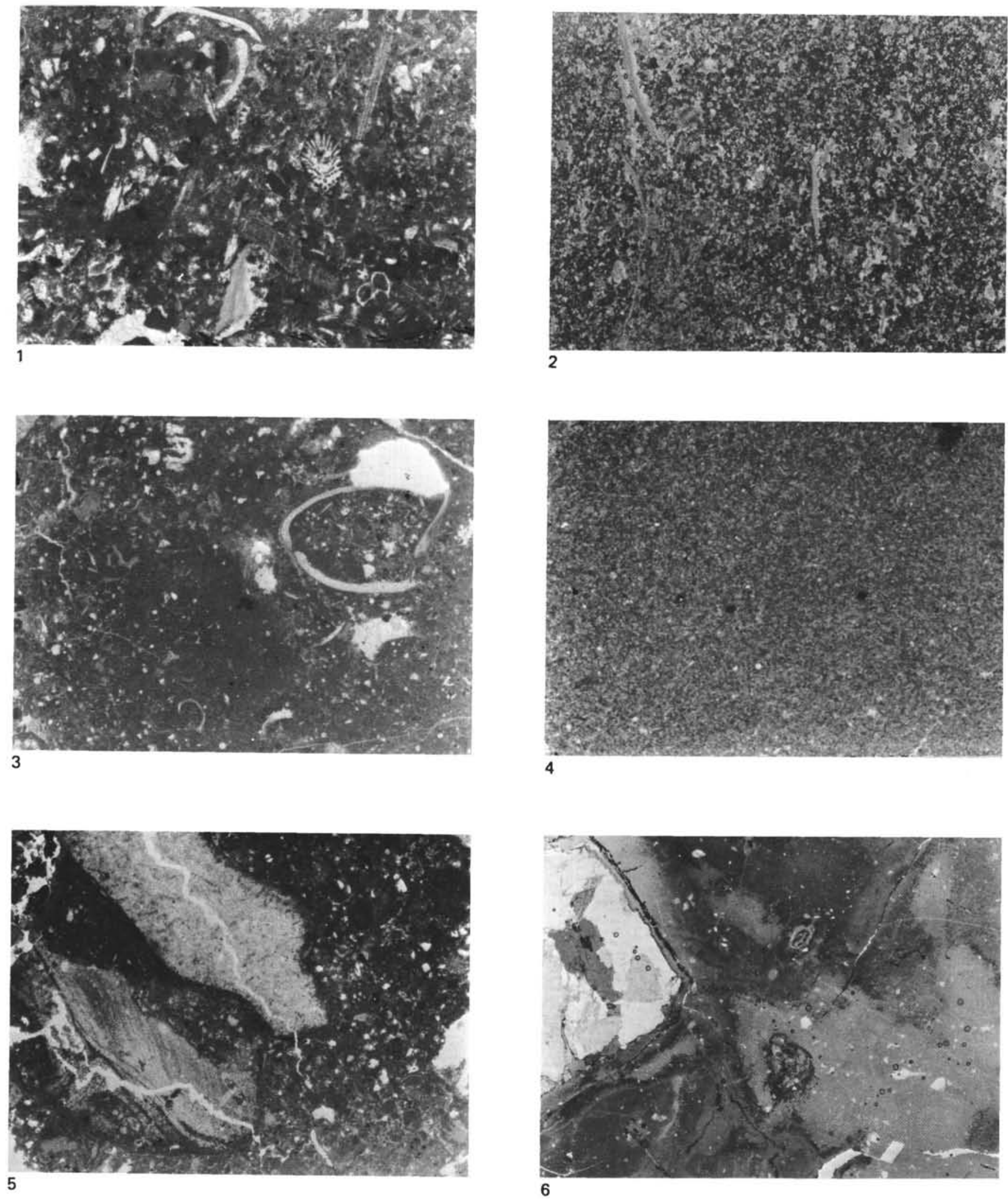


\section{PLATE 2}

Figure $1 \quad C H 67$ DR 20 C23/3 Austell Spur. Biomicrite including numerous disarticulated well-sorted Inoceramus prisms, radiolarians, planktonic foraminifers in a clayey matrix. Late Cretaceous. External platform; $15 \times$.

Figure 2 SU 01 DR 11 Gl King Arthur Castle. Calcareous breccia including shallow-water Aptian limestone clasts (Orbitolina; Echinids, algae); sandstone and clayey clasts; clayey matrix contains Eocene nannoflora. $12 \times$.

Figure 3 SU 01 DR 04 C5 Little Sole (SW). Micrite including algae, Archeolithothamnium, Dasycladacae, bryozon, Asanöna, planktonic foraminifers, echinoderms, quartz. Late Eocene to Miocene, Infratidal; $12 \times$.

Figure 4 CH 67 DR $20 \mathrm{C23/1}$ Austell Spur. Algal biomicrite, miliolidae, gastropods. Eocene. Lagoonal; $12 \times$. 
PLATE 2

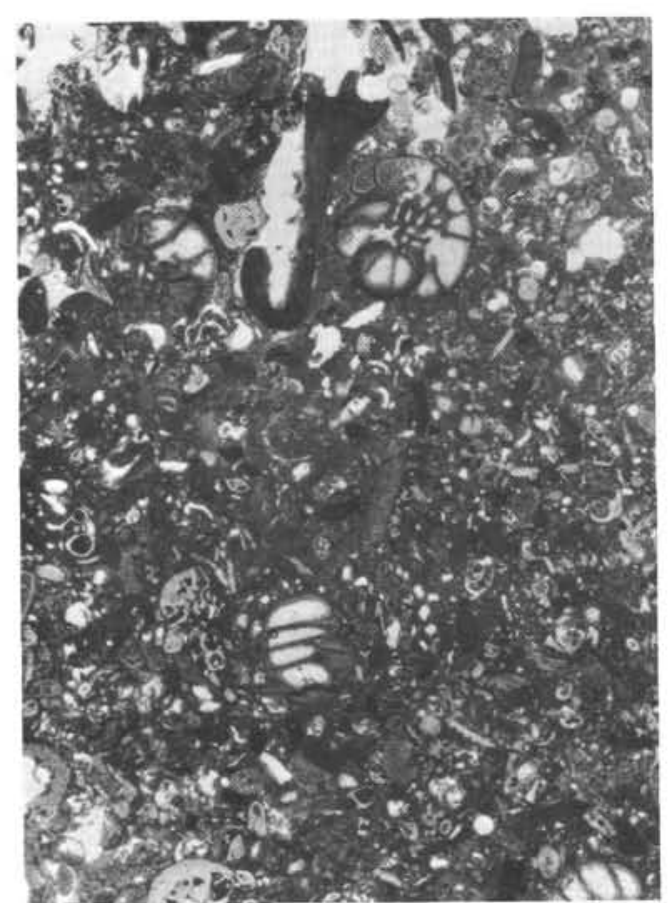

1

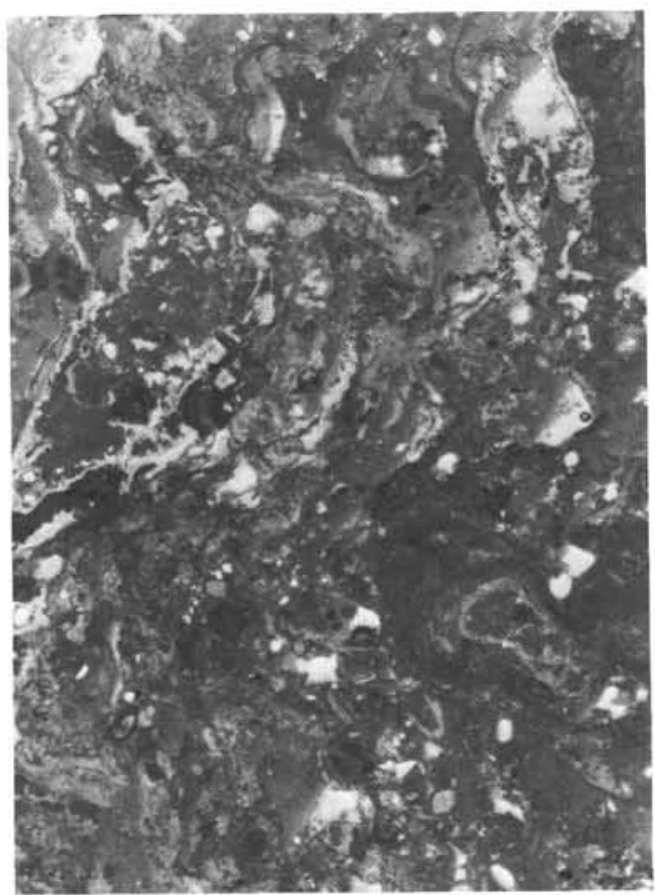

3

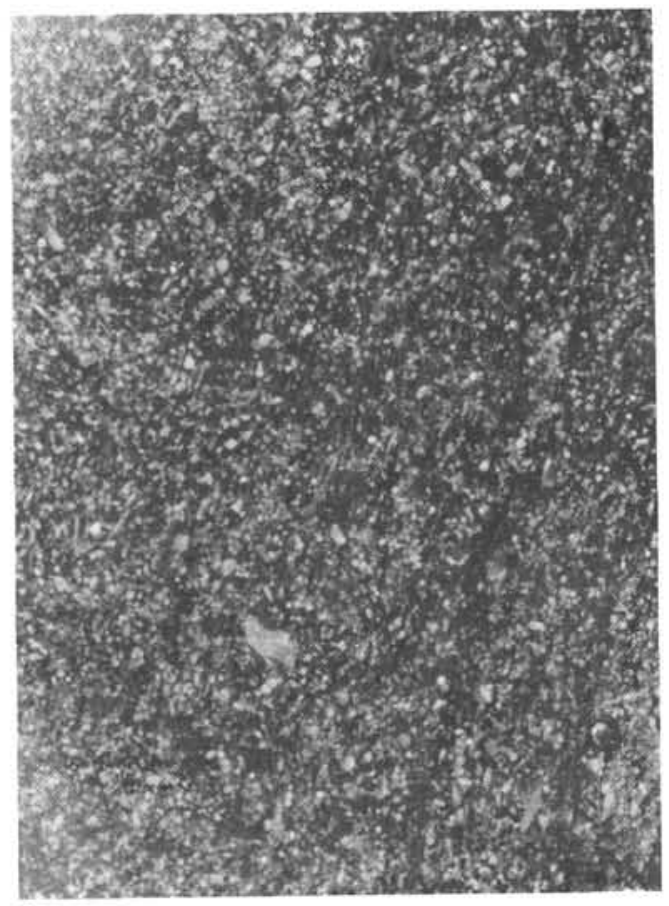

2

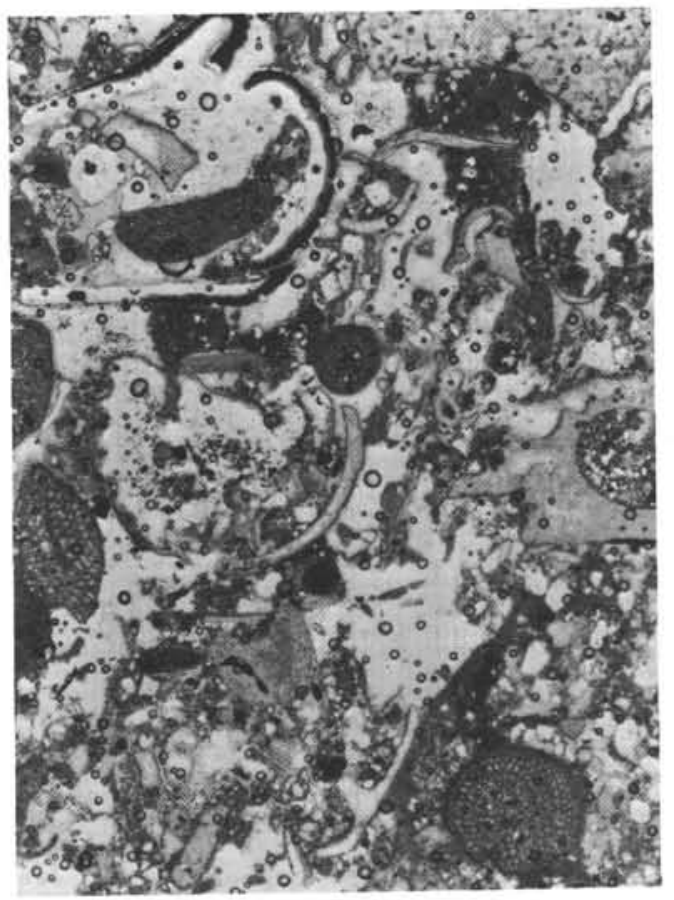

4 